Portland State University

PDXScholar

$12-20-2018$

\title{
The Effect of Styrene-Maleic Acid (SMA) Copolymers on Solubilizing Lipid Bilayers and Forming Nanodiscs
}

Ghada Alramadan

Portland State University

Follow this and additional works at: https://pdxscholar.library.pdx.edu/open_access_etds

Part of the Biophysics Commons

Let us know how access to this document benefits you.

Recommended Citation

Alramadan, Ghada, "The Effect of Styrene-Maleic Acid (SMA) Copolymers on Solubilizing Lipid Bilayers and Forming Nanodiscs" (2018). Dissertations and Theses. Paper 4843.

https://doi.org/10.15760/etd.6719

This Thesis is brought to you for free and open access. It has been accepted for inclusion in Dissertations and Theses by an authorized administrator of PDXScholar. Please contact us if we can make this document more accessible: pdxscholar@pdx.edu. 
The Effect of Styrene-Maleic Acid (SMA) Copolymers on Solubilizing Lipid Bilayers and Forming Nanodiscs

by

Ghada Alramadan

A thesis submitted in partial fulfillment of the requirements for the degree of

\author{
Master of Science \\ in \\ Physics
}

Thesis Committee:

Drake C. Mitchell, Chair

Erik J. Sanchez

Shankar B. Rananavare

Portland State University

2018 


\begin{abstract}
Cell membranes, or plasma membranes, play an essential role in the structure and the function of living cells. In 1972, the fluid mosaic membrane model was the first unifying paradigm of membrane structure. It is no longer considered adequate because evidence of many non-homogeneous lipid structures in both natural and model membranes have been discovered over the past thirty years. The field of membrane biophysics now uses updated versions of the mosaic model, which consists of the complex mixture of different lipid species. The lipid species found in natural membranes produce a range of dynamic, laterally segregated, non-homogeneous domains, which exist on time scales ranging from microseconds to minutes. The cell membrane is an enclosing or separating membrane that acts as a selectively permeable barrier within living things. It consists of the phospholipid bilayer with associated embedded proteins, integral (intrinsic) and peripheral (extrinsic) proteins used for various biological activities. Proteins, especially integral membrane proteins, perform a range of key functions vital to the cell, such as controlled movement of molecules across lipid bilayers, as well as participating in cell signaling and motility. The major obstacle to studying membrane proteins is the tendency for some of their properties to change and the proteins themselves may be denatured when extracted by detergents. One of the most significant approaches to solve this problem is the use of styrene-maleic acid copolymers (SMAs), which offers detergent-free solubilization of membrane, which allows studies of membrane proteins to be done in very small systems.
\end{abstract}


The main goal of this thesis is to examine the effects of these polymers on the interior of the lipid bilayer. With these, membrane proteins can be extracted from cell membranes while conserving a patch of near-native membrane around them. It has been suggested but not proven that proteins in nanodiscs reside in a hydrophobic environment that is identical to that found in the native cell membrane. Moreover, I also investigate the kinetics of membrane solubilization by SMA by using UV/visible spectrophotometer. In addition, I examine how lipid packing in the nanodiscs is affected by the presence of the polymers and how it depends on polymer composition by using SMA variants with different styrene-to-maleic acid ratios. 


\section{Acknowledgements}

I would like to thank my advisor Dr. Drake C. Mitchell for his assistance, enthusiasm, and guidance over the two years throughout my time as a graduate student. I would like to thank my lab mate Min Schrader for her advice and helpful discussions and assistance. I acknowledge that this thesis would have been impossible without the help of Portland State University Biophysics Lab. 


\section{Table of Contents}

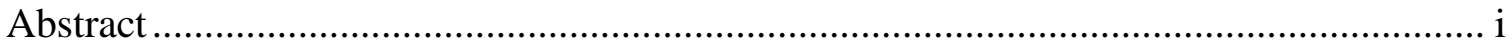

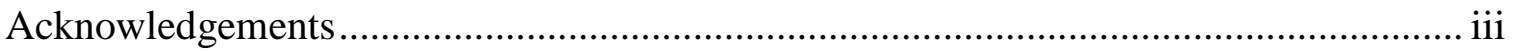



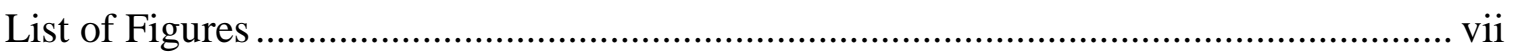

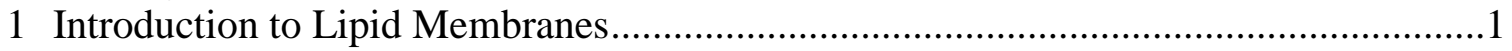

1.1 Molecular Structures of Plasma Membranes......................................................



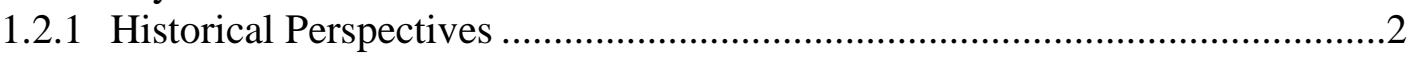

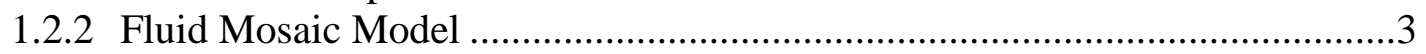

1.2.3 Lipid Raft Hypothesis ………….........................................................

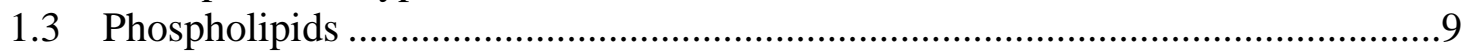

1.3.1 Micelles, Liposomes, and Bilayers..............................................................11



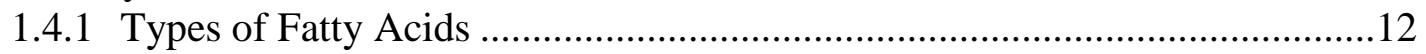

1.4.2 Monounsaturated and Polyunsaturated Fatty Acids .......................................14

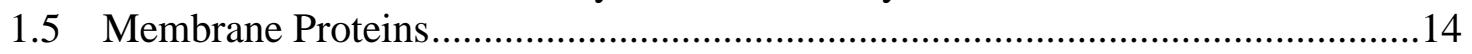

1.5.1 Brief Introduction to Proteins and Membrane Proteins....................................14

1.5.2 Studying Membrane Proteins Methods of Solubilizing ...................................16

1.5.3 Approaches Used to Solubilize Membrane Proteins ........................................17

1.5.4 Kinetics of Membrane Solubilization by SMA ……...................................24

1.5.5 Model for the Mode of Action of SMA Copolymers ...................................2.

2 Fluorescence Spectroscopy and Fluorescent Probes ....................................................28



2.2 DPH

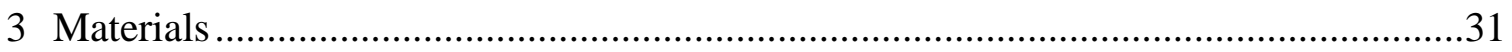

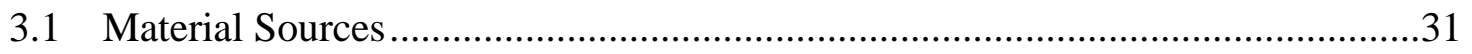

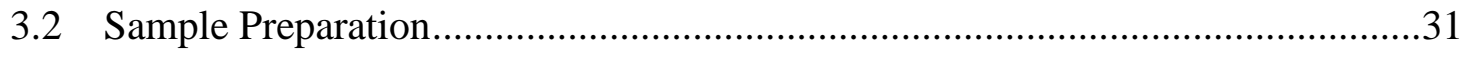



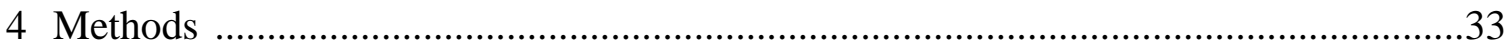

4.1 Background: Jablonski Energy Diagram.............................................................33

4.2 Definition of Absorbance and Optical Density ……….....................................34



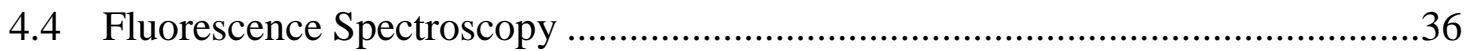



5 Frequency-Domain Lifetime and Anisotropy Measurement.........................................39

5.1 Time-resolved Fluorescence (Intensity Decays) ………….....................................39

5.2 Time-resolved Anisotropy Decays ...................................................................4

5.3 Rotational Diffusion Model...........................................................................

5.4 Least-Squares Analysis of Frequency-Domain Intensity Decays .........................46



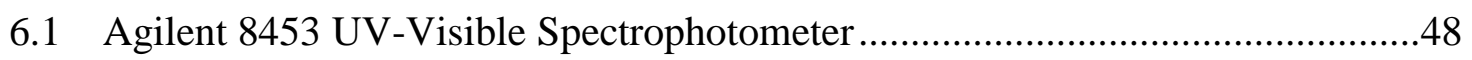

6.1.1 Fluorescence Spectra .........................................................................50

6.2 ISS Chronos Spectrometer .........................................................................52 




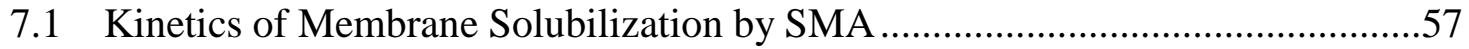

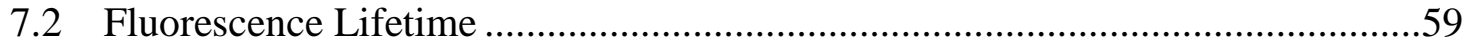



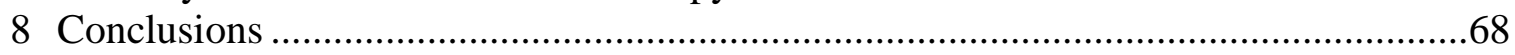

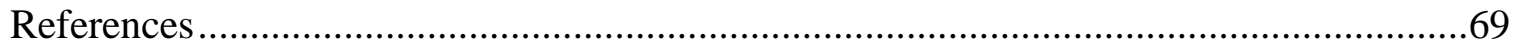




\section{List of Tables}

Table 1.1 Some Synthetic Organic Polymers ....................................................... 22 


\section{List of Figures}

Figure 1.1 From Cell Membrane to Phospholipid ..........................................................

Figure 1.2 Lipid Raft Organization Scheme …………............................................6

Figure 1.3 Effects of Unsaturated Lipids on Bilayers .....................................................

Figure 1.4 Change of Phase Behavior with Temperature ...............................................8

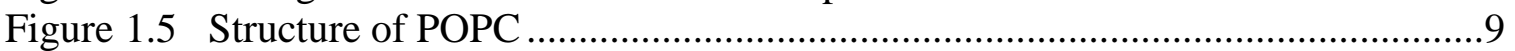

Figure 1.6 Structures of Liposomes and Micelles ..........................................................12

Figure 1.7 Acyl Chains with cis and trans Fatty Acids ...........................................13

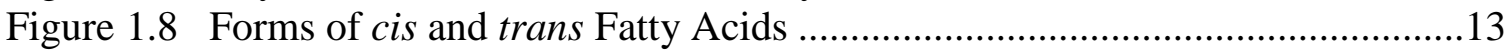

Figure 1.9 Types of Membrane Proteins …………..................................................15

Figure 1.10 Structure of Styrene-Maleic Acid (SMA) ……………………………....23

Figure 1.11 Membrane-mimetic Systems for Membrane Protein Stabilization ............... 24

Figure 2.1 Structure of Diphenylhexatriene …………............................................29

Figure 2.2 Partitioning of Fluorescent Probes in Lipid Bilayers ....................................30

Figure 3.1 Flow Chart of Production of LUVs..........................................................32

Figure 4.1 Jablonski Energy Diagram for Fluorescence...................................................33

Figure 5.1 Definition of the Phase Angle and Modulation of Emission..........................40

Figure 5.2 Frequency-Domain Measurements of Anisotropy Decay ...............................42

Figure 5.3 Example of Anisotropy Decay ...................................................................43

Figure 6.1 Optical System in Agilent 8453 UV-Visible Spectrophotometer ..................50

Figure 6.2 Absorption of Light Passing Through a Sample ...........................................51

Figure 6.3 Schematic of ChronosFD, the Frequency-Domain Fluorimeter from ISS .....52

Figure 6.4 Magic Angle ..................................................................................5

Figure 6.5 Examples of POPC Lifetime Data with or without SMA (3:1) at $25^{\circ} \mathrm{C}$........55

Figure 6.6 Examples of POPC Anisotropy Data with or without SMA $(3: 1)$ at $25^{\circ} \mathrm{C}$....56

Figure 7.1 Kinetics of Membrane Solubilization by SMA 2:1 ..................................57

Figure 7.2 Kinetics of Membrane Solubilization by SMA 3:1 ………........................58

Figure 7.3 Lifetime Change of DPH with SMA 2:1 and SMA 3:1 ……......................60

Figure 7.4 Rotational Correlation Time..................................................................61

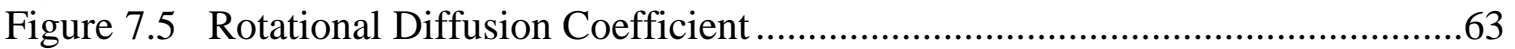

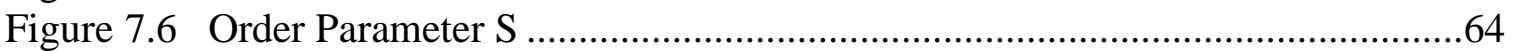

Figure 7.7 Orientational Freedom Parameter $F_{\text {rand }}\left(\right.$ Disorder Parameter $F_{\text {rand }}$ )................65

Figure 7.8 Orientation Distribution Function …………...........................................66

Figure 7.9 Orientational Probability Distribution..........................................................66 


\section{Introduction to Lipid Membranes}

\subsection{Molecular Structures of Plasma Membranes}

Biological cell membranes consist of a mix of phospholipids, which self-assemble into a two-layered sheet in aqueous media called a bilayer. Since water is the key component of living organisms, both inside and outside of the cell, interactions with water causes the phospholipids to line up in two layers with the hydrophilic heads pointing outward and the hydrophobic tails hidden in the middle and is typically 5 to 8 $\mathrm{nm}$ thick, as shown in Figure 1.1. Biological membranes are essential to both the structure and function of all living organisms; they act as a barrier between the contents inside the cell and the environment outside of the cell. The basic function of a cell membrane is to protect the cell from the surrounding environment. In addition, it

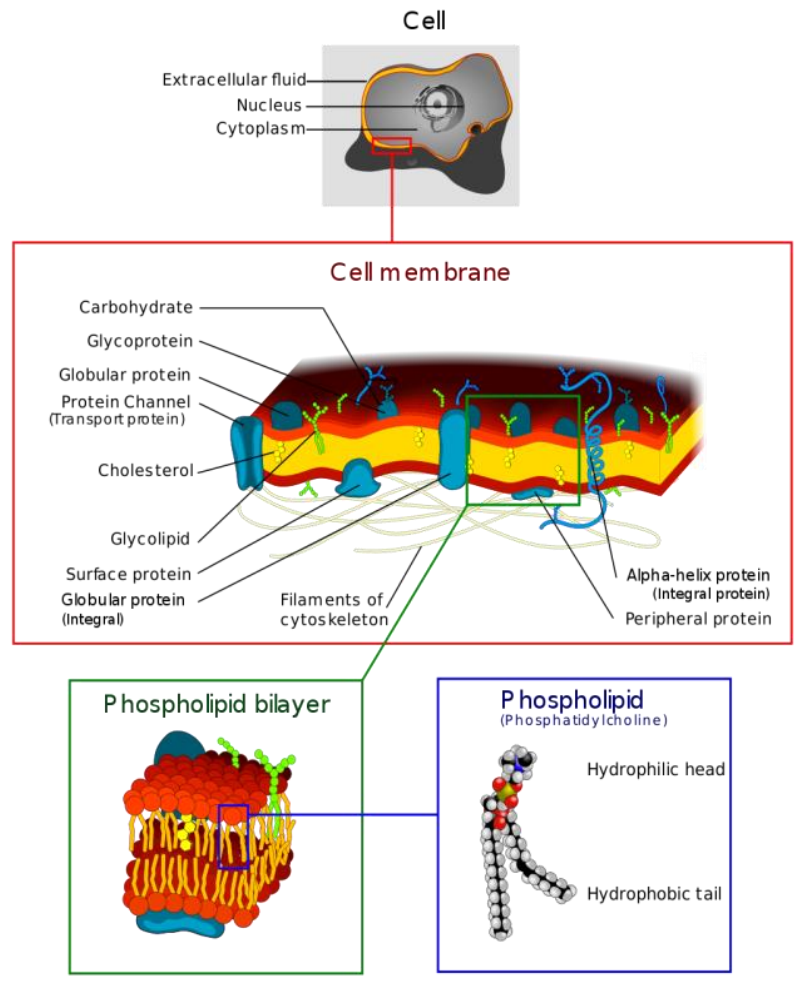

Figure 1.1: From Cell Membrane to Phospholipid [3] 
regulates molecular movement into and out of the cell. Plasma membranes typically consists of more than 1500 types of lipid species and a hundred or more types of proteins [1]. The most abundant lipids in cell membranes are phospholipids, sphingolipids, and cholesterol [2].

\subsection{History of Membrane Models}

\section{$\underline{\text { 1.2.1 Historical Perspectives }}$}

In 1895, Ernest Overton proposed that the cell membranes are distinct from the cellulose cell walls and are made of a lipid layer [4]. In 1917, the oldest artificial membranes systems, called Langmuir films, were devised. These films are monomolecular lipid films at the air-water interface [5]. Two Dutch physiologists, Gorter and Grendel, reported the first documented evidence that cell membranes are arranged in a lipid bilayer configuration in 1925 [5]. Although they arrived at the correct model of plasma membrane structure, they did not discover the proteins as components of biological membranes. In 1935, Davson and Danielli proposed that membranes have proteins. Therefore, they stated that biological membranes consisted of lipid bilayers coated by globular proteins on the outer surfaces of the lipid bilayers. Their model illustrated a "sandwich" of protein-lipid-protein [6]. By the 1950s, the improvement in electron microscopy (EM) resolution allowed Robertson to extend the Davson-Danielli model into the Davson-Danielli-Robertson (DDR) model [7]. Robertson proposed that all biological membranes share a similar underlying structure, which he called "unit membrane" with a thickness of 6 to $8 \mathrm{~nm}$. In 1964, Alec Bangham used EM and observed artificial membranes formed by phospholipids in the form of liposomes [8]. 
In 1966, the unit membrane model was challenged by observations from Green and Benson $[9,10]$. They noticed that there was a large and wide function diversity of membranes, contradicting the unit membrane model. Thus, the idea that biological membranes could be made up of specific subunits seemed appealing [4]. They demonstrated that subunits contain lipids and proteins and can be separated from the whole membrane, and these subunits can be reconstituted to regain activity. The observations by Green and Benson postulated different models where the lipids work like a solvent for embedded globular proteins $[10,11]$. EM experiments allowed experimenters to visualize the structures of frozen fractures of biological membranes and proved that the embedded proteins were clearly demonstrated in biological membranes. In addition, the idea of integral membrane proteins was introduced for the first time by these experiments. In 1972, Singer and Nicolson developed new ideas for membrane structure called fluid mosaic model, which was the dominant model until recently. Indeed, it was very important to consider all these contributions because they illustrated that membrane structures were not immediately recognized by the community working on membrane studies.

\subsubsection{Fluid Mosaic Model}

In 1972, a new model of membrane developed by S. J. Singer and Garth Nicolson, which is known as the fluid mosaic model (FMM), to describe the cell membranes structures [12]. In spite of many controversial ideas proposed in the 1970s before the FMM, it incorporated many relevant experimental facts and development of techniques for the first time [13]. One of the most significant features of the FMM is the fluidity in 
the lipid bilayer. The fluidity concept described the lipid bilayer as a pseudo twodimensional liquid in which both lipids and membrane-associated proteins can move laterally to allow for function. Thus, the membrane is more like a fluid than a solid. The Singer-Nicolson model also emphasized the mosaic nature of proteins, which could span the lipid bilayer. The overall random appearance of the biological membrane as a lipidprotein fluid composite made the membrane look like a mosaic.

The FMM has been a central description of membrane structure since the development of the freeze-fracture technique and immunoelectron microscopy. However, after forty years many non-homogeneous lipid structures have been observed in both natural and model membranes, thus, the Singer-Nicolson model is no longer considered adequate. The field of membrane biophysics now includes experimental evidence of the existence of a complex mixture of different lipid species found in natural membranes that produce a range of dynamic, laterally segregated, non-homogeneous structures, which exist on time scales ranging from microseconds to minutes.

\subsubsection{Lipid Raft Hypothesis}

Specialists in lipids research determined that lipids could laterally segregate in membranes under certain conditions of composition and temperature and form distinct lipid domains at approximately the same time the FMM was proposed [14,15]. Between 1976 and 1984, Marcelja and Sackmann proposed that the different membrane regions induced by lipid-protein interactions are the physical basis for membrane-mediated processes $[15,16]$. In 1988, Simons and van Meer [17] proposed a particular functional aspect of specialized domains called lipid rafts. Simons and Ikonen developed the lipid 
rafts idea in 1997 [18]. The lipid rafts postulated that cholesterol and sphingolipids such as sphingomyelin spontaneously associate with each other to form platforms for the segregation of proteins. The proposal of coexisting fluid phases met the requirement of high degree of mobility of a membrane and it provided a system for bilayer separation with different acyl chain orders and thickness.

Lipid rafts differ from the rest of plasma membrane in that they are more ordered and tightly packed and float freely in the membrane bilayer [19]. Lipid rafts can be isolated and extracted from the remaining plasma membrane when treated at low temperature $4^{\circ} \mathrm{C}$ with non-ionic detergents, such as Triton X-100 or Brij-98 [20].

The lipid distribution in the two leaflets of the bilayer is not random. Biological membranes have two liquid phases of separation, namely liquid ordered phase $\left(\mathrm{L}_{\mathrm{o}}\right)$ and liquid disordered phase $\left(\mathrm{L}_{\mathrm{d}}\right)$. The $\mathrm{L}_{\mathrm{o}}$ phase is a domain enriched with cholesterol and sphingolipids that appear more tightly packed and stable than the surroundings. The $\mathrm{L}_{\mathrm{d}}$ phase is less ordered than the surroundings, which are considered a highly fluid state with irregular packing of individual lipid molecules $[21,22]$. The $\mathrm{L}_{\mathrm{o}}$ and $\mathrm{L}_{\mathrm{d}}$ phases co-exist in the lipid bilayer under a certain range of temperatures, depending on the kind of lipid present. In the case where $\mathrm{L}_{d}$ is the connected phase, the lipids in the $\mathrm{L}_{\mathrm{o}}$ phase can float freely like an ice raft floating in the sea. However, the two phases can possibly coexist depending on the relative composition of the constituents, where it would be like pools of water in a plane of ice. One of the significant ways to visualize the lipid raft and describe the arrangement that contains various lipids and proteins inside the membranes is the description of the dynamic entities. 
Figure 1.2 shows the structure of the lipid bilayer sheet. Although not all of the phospholipid chains within the raft are fully saturated, the hydrophobic chains of the lipids contained in the rafts are more saturated and more tightly packed than those in the surrounding bilayer. Due to the enrichment of saturated phospholipids and cholesterol, the $\mathrm{L}_{\mathrm{o}}$ phase in the middle shown in the middle of Figure 1.2 is thicker than the surroundings.

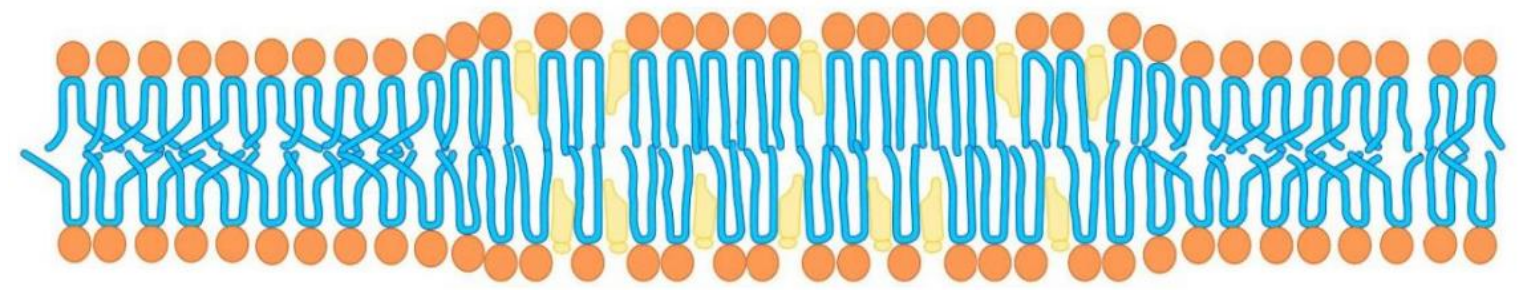

Figure 1.2: Lipid Raft Organization Scheme [23]

The phase behavior of the lipid bilayer is one of the most important properties, and is defined as a relative mobility (fluidity) of lipid molecules that change with temperature. The "melting temperature" $\left(\mathrm{T}_{\mathrm{m}}\right)$ is defined as the peak of the transition when the lipid bilayer transforms from solid phase to liquid phase. The solid phase is commonly called the "gel" phase. The phase behavior of lipid bilayers is largely determined by the strength of the attractive Van der Waals interactions between adjacent lipid molecules. Generally, the melting temperature is higher with lipids that have longer acyl chains; this because more carbon molecules have more interaction energy. Moreover, at a certain temperature, a lipid with short acyl chains (short-tailed) will be more fluid than an identical lipid with long acyl chains (long-tailed) lipid [24]. There is another factor that affects the transition temperature beside the length of the carbon chain, which is the unsaturation of the acyl chain. An extra double bond causes a kink on the 
acyl chain, which leads to extra space between acyl chains and necessitates more flexibility from the adjacent chains, as shown in Figure 1.3. Thus, increasing double bonds can also lower the transition temperature.

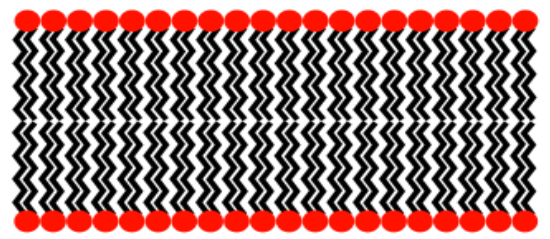

Saturated lipids only

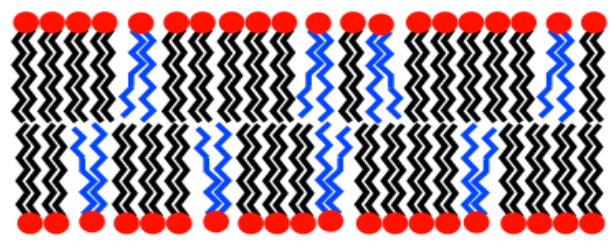

Mixed saturated and unsaturated

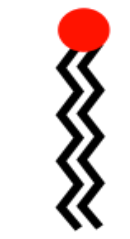

Saturated



Monounsaturated

Figure 1.3: Effects of Unsaturated Lipids on Bilayers [25]

Figure 1.4 demonstrates the phase behavior change in membranes according to temperature change. In the diagram, blue color represents the liquid-disordered phase, purple represents the liquid-ordered phase, and green color represents the solid, or gel phase. The most notable thing in this diagram between the liquid-ordered phase and liquid-disordered phase is that the acyl chains of phospholipids in the liquid-ordered phase are more ordered, or more tightly packed, and have a higher relative population of cholesterol than that in liquid-disordered phase. However, in the solid phase, the cholesterol molecules have been "squeezed out" by the crystalline structure of the acyl chains. 



Figure 1.4: Change of Phase Behavior with Temperature [26]

There is a decrease in temperature from Figure.1.4a to Figure. 1.4c. Figure 1.4a shows the lipids in liquid-disordered phase $\left(\mathrm{L}_{\mathrm{d}}\right)$ at $37^{\circ} \mathrm{C}$ (close to mammalian body temperature) where shows fast motions of lipid chains. Figure 1.4b shows the lipids at a lower temperature $\left(23^{\circ} \mathrm{C}\right.$ for example, which is approximately room temperature), where a part of a liquid-disordered phase $\left(\mathrm{L}_{\mathrm{d}}\right)$ changes to liquid-ordered phase $\left(\mathrm{L}_{\mathrm{o}}\right)$ as temperature goes down. This is because that lower temperature provides less thermal energy for lipid molecules to diffuse and exchange locations. Figure $1.4 \mathrm{c}$ shows the change from a liquid phase to a solid (gel) phase at a lower temperature $\left(10^{\circ} \mathrm{C}\right.$ for example). As previously mentioned, at some certain temperature, lipid membranes transform from liquid phase to solid phase.

Indeed, many lipids membranes have a free motion, where some of them diffuse laterally in the plane of the bilayer, others rotate about a molecular axis roughly normal to the bilayer plane, and some "flip" between the two leaflets of the lipid bilayer. Individual phospholipids also possess many degrees of conformational freedom. 


\subsection{Phospholipids}

The major lipid components in cell membranes are phospholipids. Phospholipids often make up over $50 \%$ of all lipids in plasma membranes. Most phospholipids are composed of a hydrophilic head group, which contains glycerol, phosphate, and choline (in the case of phosphatidylcholines), and a hydrophobic tail consisting of two fatty acid acyl chains. One exception is sphingomyelin, which has sphingosine instead of glycerol in head group, and a single acyl chain. In 1847, the French chemist and pharmacist Theodore Nicolas Gobley was the first to identify the phosphatidylcholine in the egg yolk as a phospholipid [27]. Phosphatidylcholines (PC) are a major constituent of cell membranes and considered as a class of phospholipids that incorporate choline as a head group. Typically, the structure of the phospholipid molecule consists of a hydrophilic head group and hydrophobic tails. The hydrophilic head group contains glycerol and a negatively charged phosphate group; the hydrophobic tails are two long fatty acid

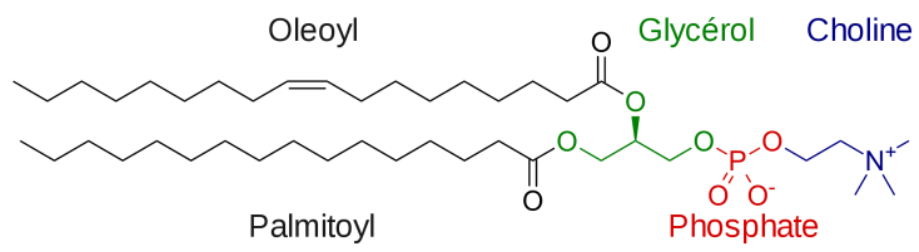

Figure 1.5: Structure of POPC [29]

hydrocarbon chains. These properties allow the phospholipid bilayer structure to be formed as a liposome in water with hydrophilic head groups facing the water both inside and outside the spherical vesicle, while the hydrophobic tails hide inside face each other inside the bilayer itself. (See Figure 1.6.) In biological systems, phospholipids form cell membrane with other molecules such as proteins, glycolipids, and sterols embedded [28] 
Figure 1.5 shows the structure of POPC (1-palmitoyl-2-oleoyl-glycero-3phospho-sn-choline), or 16:0, 18:1 PC, a type of phospholipid common in lipid bilayers. The different colors show the basic structural elements common to all phospholipids. The green part is the three-carbon glycerol backbone, which is the starting place for building the structure. To the right, the red phosphate group and blue choline group together form the hydrophilic phosphatidylcholine (PC) head group. The two hydrophobic acyl chains, shown in black, provide the "P" and "O" to the name POPC. Note that the palmitoyl chain has no double bonds, while the oleoyl chain has one double bond in the middle.

I chose POPC for my study because it is fluid at $25^{\circ} \mathrm{C}$, and it has one cis bond in an unsaturated fatty acid chain), which is typically liquid at room temperature, and the melting point of cis-double bond chains is lower than the melting point of trans unsaturated chains.

Acyl chains in phospholipids are long chains of carbon atoms with single bonds between them, and are called saturated whenall remaining carbon bond positions are saturated with hydrogen atoms. These single bonds allow free rotation about each bond. Unsaturated fatty acid chains have one or more double bonds in a chain. A double bond in a fatty acid chain restricts rotation about the bond between two neighboring carbons in the chain. The definition of a cis bond is that the carbon chain continues after the double bond on the same side as the previous section of the carbon chain, resulting in a kink, as shown in the bottom right corner of Figure 1.3. The acly chains in phospholipids usually contain an even number of carbon atoms, typically between 16 and 20 . The 16- and 18carbon fatty acids are the most common. Phosphatidylcholines with saturated acyl chains or monounsaturated acyl chains (having a single double bond) have been heavily studied. 
However, the biological function of polyunsaturated acyl chains (with multiple double bonds) abundant in the membranes of the pre-and post-synaptic membranes, retina, and spermatozoa is not yet clear. Polyunsaturated fatty acids will be further discussed in Section 1.4.

\subsubsection{Micelles, Liposomes, and Bilayers}

Lipids when added to water can form micelles or liposomes spontaneously. They both are often composed of phospholipids, which have hydrophilic head groups and hydrophobic acyl chains. Some lipids like sphingolipids have only one acyl chain, so the cross sectional area of the head group is greater than the cross section of the acyl chains, and the individual units are wedge-shaped. In this case, micelles instead of liposomes are formed (See Figure 1.6.) In aqueous solution, the hydrophilic head groups protect the hydrophobic acyl chains from the water. Structures such as dirt can be captured into the micelles. Thus, micelles widely exist in detergent applications. However, some other lipids have two acyl chains, which gives cross sectional areas of the head groups roughly equal that of the acyl chains; the individual units are cylindrical. In this case, lipid bilayers can be formed as liposomes. Liposomes can be unilamellar vesicles consisting of a single bilayer surrounding the internal aqueous compartment, or multilamellar vesicles consisting of multiple bilayers surrounding the enclosed aqueous solution. Unilamellar vesicles can be prepared from large multilamellar vesicles (LMV) which is a large "onion-like" structure consisting of multiple nested bilayers. The small unilamellar vesicles (SUV) with one lipid bilayer are typically 15 to $30 \mathrm{~nm}$ in diameter. Large unilamellar vesicles (LUVs) range from 100 to $200 \mathrm{~nm}$ or larger [30], and the size of 
giant unilamellar vesicles (GUV) is on the order of a few tens of micrometers, which is similar to the size of the plasma membrane of cells. Figure 1.6 shows the structures of two three-dimensional structures of micelle and liposome. I prepared unilamellar vesicles from multilamellar vesicles by extruding a suspension of MLVs through $200 \mathrm{~nm}$ diameter pores.

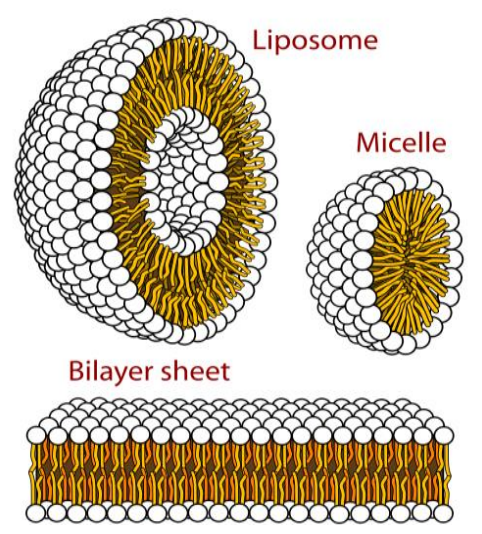

Figure 1.6: Structures of Liposomes and Micelles [31]

\subsection{Fatty Acids}

\subsubsection{Types of fatty acids}

Fatty acids are long chain hydrocarbons possessing a carboxyl $(\mathrm{COOH})$ group at one end. Natural fatty acids have an unbranched chain of an even number of carbon atoms, from 4 to 28 . If the fatty acids have no double bond, they are called saturated. If the fatty acids do have double bonds, they are called unsaturated. An unsaturated fatty acid has with one double bond within the fatty acid chain is called monounsaturated fatty acid, and if it contains more than one double bond, it is called a polyunsaturated fatty acid. 

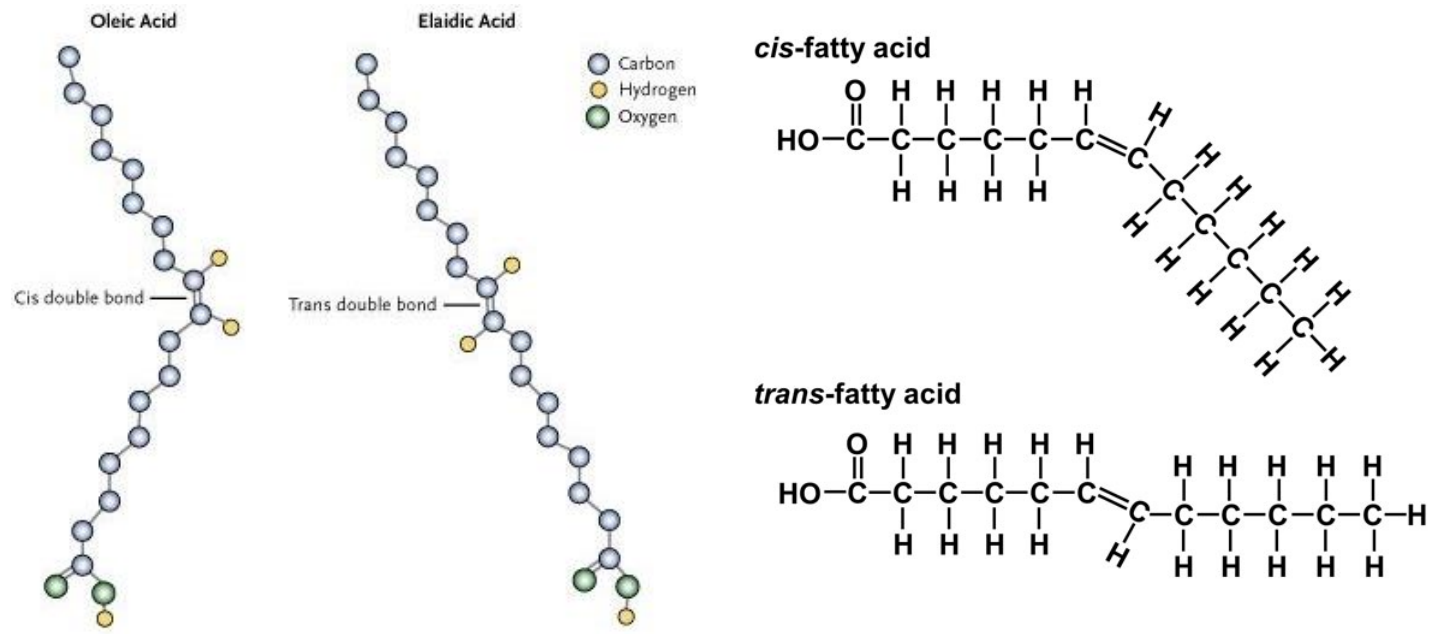

Figure 1.7: Acyl Chains with cis and trans Fatty Acids [32, 33]

There are two forms of double bonds in unsaturated fatty acids, one is called trans, and the other is called cis. In cis fatty acids, the ends of the hydrocarbon chain are on the same side of the double bonds, while in trans fatty acids, the ends of the hydrocarbon chain are on the opposite side of the double bond. The difference between these two types of double bonds will affect the shape of the fatty acids, as shown in Figure 1.7. The double bonds in cis fatty acids lead to a bent form of the acyl chain, whereas trans double bonds lead to a straight form of the acyl chain as shown in Figure 1.8 .



Figure 1.8: Forms of cis and trans Fatty Acids [34] 


\subsubsection{Monounsaturated and Polyunsaturated Fatty Acids}

Monounsaturated fatty acids (MUFA) contain one carbon-carbon double bond. MUFA can be found in red meat, whole milk products, nuts, and high fat fruits such as olives and avocados. Common monounsaturated fatty acids are palmitoleic acid (16:1 $n-7)$ and oleic acid (18:1 n-9). However, polyunsaturated fatty acids (PUFA) contain two or more carbon-carbon double bonds. Polyunsaturated fatty acids can be found in seeds, fish that include many important compounds labeled essential fatty acids (EFA). Essential fatty acids can only be ingested from food and cannot be synthesized in the human body. Only two EFAs are known for humans: alpha-linolenic acid (an omega-3 fatty acid) and linoleic acid (an omega-6 fatty acid) [35].

\subsection{Membrane Proteins}

\section{$\underline{\text { 1.5.1 Brief Introduction to Proteins and Membrane Proteins }}$}

Biological membranes are lipid bilayers that provide the basic structure of cell membranes, however, they also include membrane proteins that perform most of the membrane's specific tasks and therefore give each type of cell membrane its characteristic functional properties. Proteins are large bio-molecules consisting of one or more long chains of amino acid residues. Proteins are differentiated by their sequences of amino acids, which result in protein folding into a specific three-dimensional structure that determines its activity. A linear chain of amino acid residues is called a polypeptide. A protein contains at least one long polypeptide. Short polypeptides, containing less than 20-30 residues, are rarely considered to be proteins and are commonly called peptides. Adjacent amino acid residues and peptide bonds bond the single amino acid residues together. The sequence of a gene is considered the key element to define the sequence of 
amino acid residues in a protein, which is encoded in the genetic code. There are four distinct aspects of protein structure; the most common one is the secondary structure, such as $\alpha$-helix and $\beta$-sheet [36].

The cell membrane has a large protein content, typically around $50 \%$ of membrane volume. Proteins are very important for cell membranes because they are responsible for various biological activities such as communication and transportation of chemicals and ions. Membrane proteins are proteins that interact entirely or partly with a biological membrane. Membrane proteins are amphiphilic, having hydrophilic and hydrophobic regions. Proteins can be associated with the lipid bilayer of a cell membrane in different ways. Membrane proteins consist of three main types: integral proteins, peripheral proteins, and lipid-anchored proteins as shown in Figure 1.9. Integral proteins extend through the lipid membrane, hence are called transmembrane proteins. Peripheral proteins interact temporarily with a biological membrane. They attach to integral membrane proteins or associate with peripheral regions of the lipid bilayer. Lipidanchored proteins are located on the surface of the cell membrane and are covalently bound to single or multiple lipid molecules. Lipid-anchored proteins insert into the cell membrane and take a place alongside the hydrophobic fatty acid tails, and anchor the protein to the cell membrane. The protein itself is not in contact with the membrane.



Figure 1.9: Types of Membrane Proteins [37] 


\subsubsection{Studying Membrane Proteins and Methods of Solubilizing}

Studying membrane proteins is very important due to their crucial roles in cellular processes, control of fundamental biochemical processes, and they are often important pharmacological drug targets [38]. However, studying membrane proteins is challenging because the process of extracting, purifying, and homogenizing proteins removes them from their native environment. Extraction from the native environment mean a serious loss of information about the proteins [39]. The best way to study protein membranes is to produce a new method mimicking the native environment of the membrane. About 20 to $30 \%$ of the proteomes of most organisms structures have been resolved by X-ray crystallography or NMR (nuclear magnetic resonance spectroscopy) [40]. In 1985, the first membrane protein structure was published when it was solved by X-ray crystallography, and since then the number has increased [40]. There are now more than 300 unique membrane protein structures discovered using the same method. In 2014, the Protein Data Bank (PDB) repository of protein structures had 148 unique proteins from 368 of the membrane proteins 3D structure [40]. Indeed, membrane proteins are embedded into biological membranes that are an anisotropic environment established by a bilayer of amphipathic lipids with a hydrophobic core that shields the hydrophobic surface of the proteins from the aqueous phase. Thus, membrane proteins need to be isolated from their complex environment while maintaining both their stability and activity. There are new methodologies for improved membrane proteins solubilization and stabilization. Finding an environment with optimal properties to allow studying the structural and functional properties of the protein, and allowing stabilization and 
purification of the protein while the protein displays native behavior is very challenging. However, various approaches of the membrane-mimetic systems are now commonly used in membrane protein research. One of the approaches is using the detergents for solubilization into micelles, and replacement of detergent by more stabilizing agents, such as amphipols. Another approach is bicelles and lipid vesicles which membrane proteins can be reconstituted into a lipid bilayer-forming environment, or by using nanodiscs that are stabilized by membrane scaffold proteins (MSPs). Recently, a new alternative approach is developed which is the use of non-detergent SMA copolymers that directly solubilize membranes in the form of nanodiscs.

\subsubsection{Approaches Used to Solubilize Membrane Proteins}

\subsubsection{Detergents}

One of the common strategies of membrane proteins isolation is the solubilization of the lipid bilayer with detergents, which generally leads to the formation of spherical micelles, comprising membrane proteins, detergent molecules, and possibly some remaining lipids. Detergents are amphiphilic compounds, which have a hydrophilic head group and one hydrophobic tail. This structure of detergent gives the detergent the ability to participate in specific biological and biochemical functions. Lipids and detergents are both amphipathic with a hydrophilic head, and hydrophobic tails, but they differ from in the shape, and in the type of aggregates formed in solution. A wide range of detergents with high solubilizing efficiency has been used to extract different membrane proteins from biological membranes with varying properties, and these detergents assisted the understanding of membrane proteins. However, detergents have some inherent 
drawbacks. First, membrane proteins are different in their properties, thus, the detergent that works for one membrane protein may not be suitable for other membrane protein. Therefore, working with membrane proteins with unknown properties requires an extensive, mainly empirical screening to find a suitable detergent (mix) for each specific case. Second, addition of detergent will remove the protein from its native lipid environment and thus, it will lead to loss of native interactions with both lipids and other proteins. Third, the solubilization of membrane proteins by detergent does not keep the native structure and stability; thus, the detergent that used for extraction may not be compatible with purification and biochemical studies. Fourth, detergent micelles are very poor mimic of lipid bilayers, because micelles have a single hydrophilic surface that is highly curved and hydrophobic parts that have a low degree of order. Furthermore, water permeability and lateral pressure profiles differ extensively in micelles and bilayers, and as a result, membrane proteins show a lower stability in micelles and transient solvent exposure of the hydrophobic membrane proteins surface can lead to inactivation or aggregation of the protein.

\subsubsection{Amphipols}

Amphipols are a new class developed by Popot and coworkers, which used as an

alternative to detergents to achieve a more stabilizing environment for membrane proteins [41]. This new class of polymers are surfactants) that possibly handle membrane proteins in detergent-free aqueous solution as though they were soluble proteins. Amphipols are amphiphilic polymers designed to extract membrane proteins and keep 
them soluble in water with the absence of detergents, but amphipols tend to be denaturing.

\subsubsection{Vesicles and Bicelles}

The real environment of the lipid bilayer is important because its physicochemical properties are considered essential for maintaining the structure, function, and stability of membrane proteins. The mimetic systems of biological membranes discussed above have a downside that they are lack of an actual lipid bilayer environment. This problem is overcome by reconstitution of membrane proteins into systems of synthetic lipids such as planar lipid bilayers or lipid vesicles.

These systems allow a systematic investigation of the effect of the membrane lipid composition on structural and functional properties of membrane proteins. However, these systems have some disadvantages, the planar lipid bilayers are immobilized systems, thus, they not suitable for solution-based methods. Vesicles have a large size that may impede optical spectroscopy due to light scattering.

Bicelles are an alternative to vesicles. Bicelles are usually obtained by mixing short-chain phospholipids with detergents in a defined ratio. They have discoidal structures and have different sizes ranging from $8-50 \mathrm{~nm}$ in diameter depending on their composition [41]. However, bicelles have some drawbacks; they are limited to certain lipid compositions and often have a problem with stability. 


\subsubsection{Nanodiscs Bounded by Membrane Scaffold Proteins}

A new approach was developed to solve some of the challenge approaches mentioned above. Nanodisc technology was designed by Slinger and coworkers as a method to transfer membrane proteins from detergent micelles into lipid nanodiscs, which are small patches of a lipid bilayer, bounded by membrane scaffold proteins (MSPs) [41]. They engineered an encircling amphipathic helical protein belt derived from human apolipoprotein A-1. An A-1 serves to shield the hydrophobic core of the lipids from the aqueous phase, therefore, MSPs are truncated forms of apolipoprotein (apo) A-I, which wrap around a patch of a lipid bilayer to form a disc-like particle or nanodisc. Membrane scaffold proteins provide a hydrophobic surface facing the lipids, and a hydrophilic surface at the outside, thus, the resultant nanodiscs keep membrane proteins soluble in aqueous solution, and provide a native-like phospholipid bilayer environment that provides stability and functional requirements of the incorporated target. Once assembled into nanodiscs, membrane proteins can be kept in solution in the absence of detergents. The diameter of nanodiscs is around $\sim 10 \mathrm{~nm}$, but there is a generation of MSP variants allows the formation of smaller $(\sim 6-7 \mathrm{~nm})$ and larger $(16-17 \mathrm{~nm})$ nanodiscs.

\subsubsection{Nanodiscs Bounded by Styrene-Maleic Acid Copolymers}

All the previous systems have one common disadvantage that is the requirement of detergents to extract native membrane proteins from biological membranes. The destabilization of transient proteins by detergents is a persistent problem for membrane protein reconstitution into both membrane-mimetic and bilayer systems. Therefore, alternative approaches were developed. Examples of these approaches includes cell-free 
protein production, MP-enriched cell-derived extracellular vesicles, genetic engineering of the MP by fusion or minimization of the exposure time with detergent. However, there is a new promising method as an alternative to detergent extraction, which is using styrene-maleic acid copolymers (SMA).

Polymers are many repetitions of one or more molecules linked together into large molecules called macromolecules. These single molecules are known as monomers and are relatively light and simple molecules. Classification of polymers depends on the repetition or variety of monomers involved in the structure. Polymers are called homopolymers when polymers are formed by the same monomer throughout the chain. When there are at least two different species of monomer in the chain, they are called copolymers. There are three types of polymers based on their source of origin: natural, synthetic, and semi-synthetic. (1) Natural polymers occur in nature and existi in natural sources like plants and animals. Examples of natural polymers are DNA, cellulose, proteins, silk, and rubber. (2) Synthetic polymers are artificially made (synthesized) by humans in the lab by a process called polymerization. The eight most common examples of synthetic organic polymers are low-density polyethylene (LDPE), high-density polyethylene (HDPE), polypropylene (PP), polyvinyl chloride (PVC), polystyrene (PS), nylon, Teflon, and thermoplastic polyurethane (TPU). (3) Table 1.1 shows some synthetic organic polymers. Semi-Synthetic polymers that are made by chemically treating a natural polymer, such as vulcanized rubber and cellulose acetate (rayon). 


\begin{tabular}{|c|c|c|c|}
\hline Monomer & Monomer Name & Polymer & Polymer Name \\
\hline & Ethylene & & Polyethylene \\
\hline & Propylene & & Polypropylene \\
\hline & Styrene & & Polystyrene \\
\hline & Tetrafluoroethylene & & Polytetrafluoroethylene \\
\hline & & & \\
\hline & & & \\
\hline
\end{tabular}

Table 1.1: Some Synthetic Organic Polymers

Styrene-maleic acid (SMA) is a copolymer consists of hydrophobic styrene and hydrophilic maleic acid monomers units, and its structure is shown in Figure 1.10. SMA extracts proteins in the form of a lipid/protein nanodisc, unlike detergents that tend to strip away most or all of the lipids in the immediate environment of a membrane protein [42]. Addition of the polymer to synthetic or biological lipid membranes leads to the spontaneous formation of discoidal particles with diameters size in the range of $\sim 10-30$ $\mathrm{nm}$. However, the disk-shaped particles are formed into different sizes depending on the shape and diameter of the embedded proteins, polymer composition, and the 
polymer/lipid ratio [43]. Surprisingly, this system shows the possibility to directly extract MPs from cells without an intermediate step of conventional detergent solubilization. In this new type of nanodiscs bounded by the polymer, the bilayer organization of the incorporated lipid molecules is conserved. Thus, the native nanodisc system combines the power of solubilization similar to detergents with the small particle size of nanodiscs, while conserving a minimally perturbed native lipid environment that stabilizes the protein.

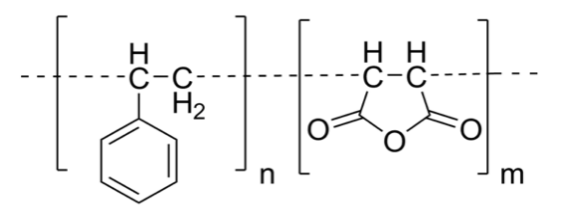

Figure 1.10: Structure of Styrene-Maleic Acid (SMA) [72]

SMA is the hydrolyzed form of the styrene-maleic anhydride (SMAnh) copolymer, which is synthesized by the copolymerization of styrene and maleic anhydride monomers. Both forms of polymers are widely used in industry fields and have different applications. For instance, the SMAnh is commonly used as thermal stabilizer in plastic blends, while SMA can be used as a dispersing agent for ink formulations and coatings. 


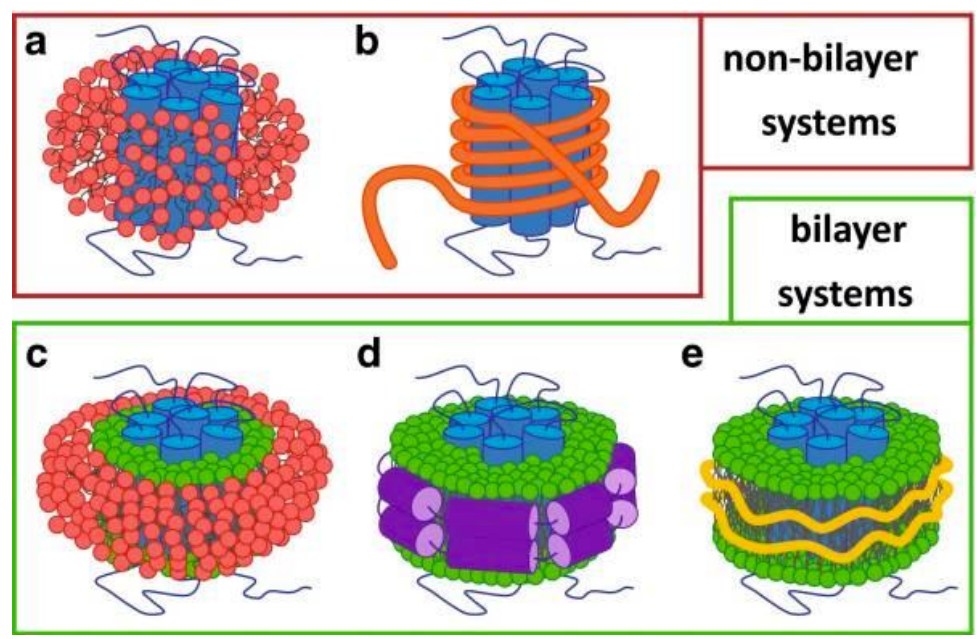

Figure 1.11: Membrane-mimetic systems for membrane protein stabilization with blue proteins and green bilayer lipids. a.) Protein in a detergent (red) micelle. b.) Protein stabilized by an amphipol (orange). c.) Protein in bicelle (detergent in red). d.) Protein in nanodisc stabilized by MSP (purple). e.) Protein in nanodisc stabilized by SMA (yellow) [41].

\section{$\underline{15.4 \text { Kinetics of Membrane Solubilization by SMA }}$}

Many studies [44] have focused on the physico-chemical characterization of discoidal particles produced by SMA polymers [44]. These particles have been referred to as SMA-lipid particles (SMALPs), lipodisq particles, or native nanodiscs. The properties of SMALPs have been studied by using different biophysical techniques. The size of the nanodiscs has been analyzed by many approaches, such as electron microscopy, which is the most common method, size exclusion chromatography, and dynamic light scattering (DLS). However, the reported size is on the order of $10 \mathrm{~nm}$ with minor variations. Indeed, the formation of SMA-bounded nanodiscs requires the solubilization of lipid membranes by the polymer. Scientists found that the simple and convenient way to monitor the kinetics of this process is turbidimetry. The solubilization process can be monitored as a decrease in light scattering over time by using a spectrophotometer, where the lipid vesicles are large particles (hundreds of nanometers 
up to micrometers in size) and thus efficiently scatter UV light, whereas SMALPs are much smaller and scatter almost no light.

\subsubsection{Model for the Mode of Action of SMA Copolymers}

It was found that many different physical parameters such as lipid composition, presence of salt, or SMA concentration affect membrane solubilization by SMA and a three-step model was developed to describe its mode of action [41]. The first step is membrane binding where SMA copolymers bind to the surface of the lipid bilayer. In our study, binding depends on the SMA concentration. It is modulated by electrostatic interactions between SMA copolymers and zwitterionic lipids at the lipid bilayer surface. The presence of zwitterionic lipids causes repulsion and thus impairs binding of the negatively charged polymer, while increasing the ionic strength promotes binding. Studies indicated that the polymers are able to insert even at surface pressures far above those estimated for biological membranes, suggesting that SMA copolymers will insert into any biological membrane [44]. The driving force for the interaction of SMA with membranes is the hydrophobic effect via the polymer styrene moieties and the lipid acyl chains, and this force is sufficiently strong to overcome any repulsive electrostatic interactions. Nevertheless, electrostatic repulsion does modulate the extent of binding, as

illustrated by the effects of increasing the salt concentration or decreasing the amount of anionic lipids in the membrane [44]. Both changes lead to increased binding, and hence to an increased efficiency of solubilization in the next step of the process.

The second step is SMA insertion and destabilization of the bilayer. In this process, SMA copolymer inserts more deeply into the hydrophobic core of the 
membrane. This is strongly affected by lipid packing (e.g., membrane fluidity and lateral pressure) and bilayer thickness. They envision that SMA will need packing defects in the membrane for efficient insertion. This is supported by the observation that in all systems of saturated lipids, maximal solubilization of SMA occurs at $\mathrm{T}_{\mathrm{m}}$ of the lipids, where gelphase domains coexist with domains of lipids in the liquid-crystalline phase, leading to large packing defects [44]. However, also in the absence of large packing defects, lipid packing is important for solubilization, as evident from the observation that at temperatures above $T_{m}$, solubilization takes place much faster than at temperatures below $\mathrm{T}_{\mathrm{m}}$, when the lipids are in the gel phase and are thus more tightly packed [44]. Interestingly, unsaturated lipids were found to be more difficult to solubilize than saturated lipids in the fluid phase. At first glance, this is surprising because unsaturated chains are more disordered and thus are more loosely packed. On the other hand, due to their double bonds, these membranes have increased lateral pressure in the acyl-chain region, and this might lead to a less efficient insertion of the polymers. In most cases, solubilization was improved by increasing temperature. This can be expected because increasing temperature will lead to a general increase in reaction kinetics and hence to faster solubilization [44]. In this study, solubilization happened at $25^{\circ} \mathrm{C}$, which is above the transition temperature of POPC $\left(-2^{\circ} \mathrm{C}\right)$ and this agrees with Scheidelaar, et al [44]. In addition, the bilayer thickness plays an important role in the solubilization process especially at the stage where the vesicles disintegrate and fall apart into intermediate vesicular structures and membrane fragments [44]. This is simply because it will be more difficult to break up thicker membranes due to an increase of the forces that hold the membrane together, such as van der Waals interactions and the hydrophobic effect. This 
is observed with short-chained saturated lipids are easily solubilized even in the gel phase, and that unsaturated lipids at lower temperature are less efficiently solubilized when the effective length of the acyl chains increases [44]. Together, these results show that the rate and yield of solubilization by SMA copolymers depends greatly on the lipid composition of the model membranes [44].

The last step is the actual solubilization of the bilayer and the simultaneous formation of nanodiscs. In this step, membrane fragments are further solubilized and the formation of nanodiscs is facilitated. It was shown that nanodiscs are indeed shaped like a disk and that the SMA copolymer places its phenyl groups between the lipid acyl chains, thereby stabilizing the nanodiscs [44]. The reported disk-like shape and the average diameter of the nanodiscs are completely independent of lipid chain length and unsaturation [44]. However, the parameters that control this nanodisk diameter are still unclear where some studies are indicating that the nanodisc diameter for SMA 2:1 is 10 $\mathrm{nm}$ and for SMA 3:1 is $30 \mathrm{~nm}$ [71]. 


\section{Fluorescence Spectroscopy and Fluorescent Probes}

\subsection{Background of Fluorescence}

In the biological system, luminescence phenomena (fluorescence) is an amazing phenomenon in nature. It can occur unexpectedly in a myriad of natural objects and systems. However, this discovery had a significant impact on scientific research. The use has been developed into the basis for analytical techniques such as UV/vis, infrared spectroscopy, and for the visualization of the microscopic scale such as in superresolution microscopy. Robert Hooke and Antonie van Leeuwenhoek were scientists who first developed optical microscopy for the observation of plant and animal cells. Since then, many scientists were involved in the development of microscopes, from white light microscopy to fluorescence microscopy [46]. However, understanding the details of molecular arrangement within different phases in biological membranes was first successfully done using nuclear magnetic resonance (NMR) spectroscopy, but this approach does not provide enough information about the different physical forms of the phases or domains $[47,48]$. By using fluorescence microscopy and incorporating probes, we can directly visualize the raft domains and report on domain shape and size. The use of fluorescence probes has been continually increased due to their versatility, sensitivity, and quantitative capabilities. There are a number of fluorescent membrane probes for membrane studies such as nitrobenzoxadiazole (NBD), rhodamine (Rhod), and diphenylhexatriene (DPH) that are used in this study. 


\subsection{DPH}

Diphenylhexatriene (DPH), or 1,6-Diphenyl-1,3,5-hexatriene, was first introduced and used by two scientists, Shinitzky and Barneholz, as a fluidity probe for membranes and bilayers, and since then it has become widely used in fluorescence anisotropy measurement [49]. DPH is not fluorescent in water, and its structure is shown in Figure 2.1. The orientation of DPH inside the lipid bilayers is loosely constrained, and is assumed to be oriented parallel to the lipid acyl chain axis, but it can also reside in the center of the lipid bilayer midplane parallel to the surface. The properties of DPH make it an ideal probe to study phase separation in lipid bilayers, especially the hydrophobic bilayer core, because DPH shows no partition preference between coexisting phases in phospholipids. Figure 2.2 shows how the fluorescent probes are incorporated into a lipid bilayer. NBD and Rhod are attached in a synthesis lab to the phosphoethanolomine (PE) head groups of lipids to form NBD-PE.and Rhod-PE. We incorporate these probes into our lipid mixtures before forming the final vesicles. DPH is also incorporated into the lipid mixture after the LUVs were formed, and usually sits between the acyl chains or between the layers in the midplane. Typically, a lipid bilayer is $5 \mathrm{~nm}$ thick, while DPH is about $1 \mathrm{~nm}$ long, thus, the length of DPH is quite comparable with the length of acyl chains.

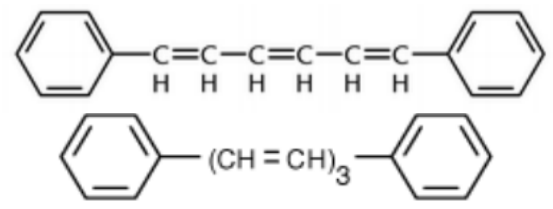

Figure 2.1: Structure of Diphenylhexatriene [50] 


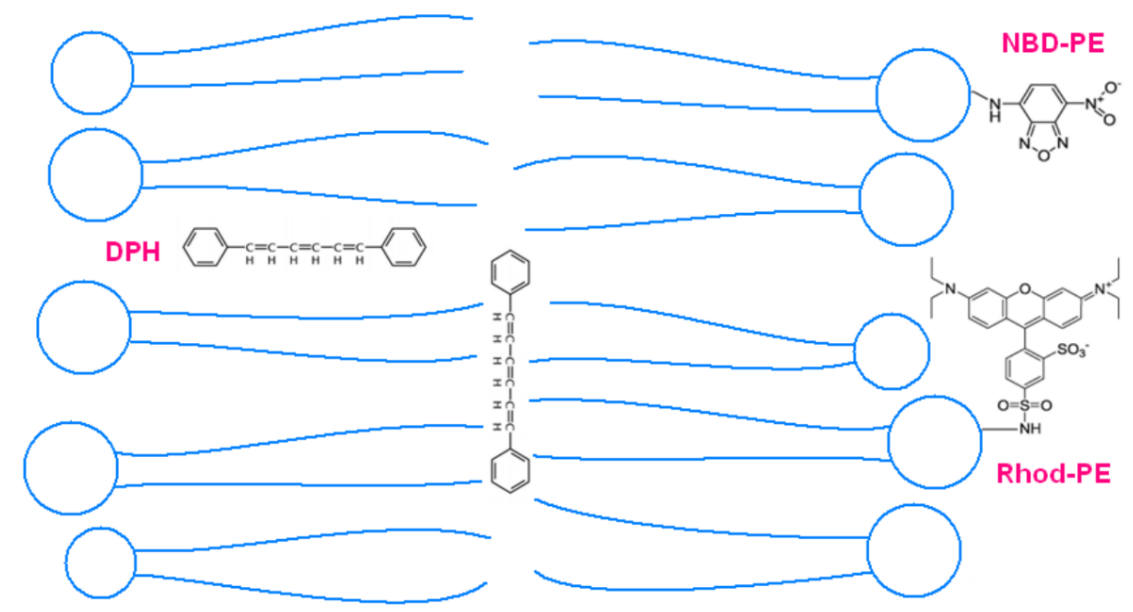

Figure 2.2: Partitioning of Fluorescent Probes in Lipid Bilayers 


\section{Materials}

\subsection{Material Sources}

The phospholipid 1-palmitoyl-2-oleoyl-sn-glycero-3-phosphocholine or 16:0-18:1 PC (POPC) was purchased from Avanti Polar Lipids (Alabaster, AL) and used without further purification. The fluorescently labeled phospholipid diphenylhexatriene (DPH) was purchased from Molecular Probes (Eugene, OR), and stored at $-20^{\circ} \mathrm{C}$. Phospholipids were stored in chloroform when manufactured. The SMA copolymers, Lipodisq 2:1 and 3:1 were purchased from Sigma-Aldrich, St Louis, MO.

\subsection{Sample Preparation}

\section{$\underline{3.2 .1 \text { Vesicles }}$}

Large unilamellar vesicles (LUV) were prepared as follows, as shown in Figure 3.1. The chloroform was driven off lipid stock solutions by rotating the vial under a stream of nitrogen gas until only a thin film of lipid coated the glass vial's surface. The resulting thin phospholipid film was dissolved by adding cyclohexane to the vial. The cyclohexane solution was frozen to a solid phase for lyophilization by putting it in a $40^{\circ} \mathrm{C}$ freezer for one hour or it was frozen by putting the vial in a beaker of dry ice and acetone for 5 minutes. Immediately after removing the vial, the lid was slightly loosened, and the vial was placed in a pump-vacuumed lyophilization chamber for at least three hours to completely remove the frozen cyclohexane. This yielded phospholipid in the form of a dispersed white powder. This powder was dissolved by adding HEPES buffer $(\mathrm{pH} 7.22)$ to the vial, and the vial was vortexed thoroughly. The contents were then divided into several smaller portions and frozen at $-20^{\circ} \mathrm{C}$. Later, each sample underwent 
through ten freeze and thaw cycles to produce multilamellar vesicles. The large unilamellar vesicles were reduced in size and formed by extruding them ten times through a pair of $0.2 \mu \mathrm{m}$ membranes by using Avanti Mini-Extruder (Alabaster, AL). Then the vesicles were mixed with HEPES buffer $(\mathrm{pH} 7.22)$ in each cuvette for the desired concentration.

Fluorescent probes are added to the lipid mixture at different times in the sample preparation process depending on the structure of the probe. DPH was added into the lipid mixture in the cuvette because DPH embeds itself between lipid acyl chains or between the two leaflets and it does not participate in the formation of vesicles. DPH was at the molar ratio of 300:1 lipid:DPH.

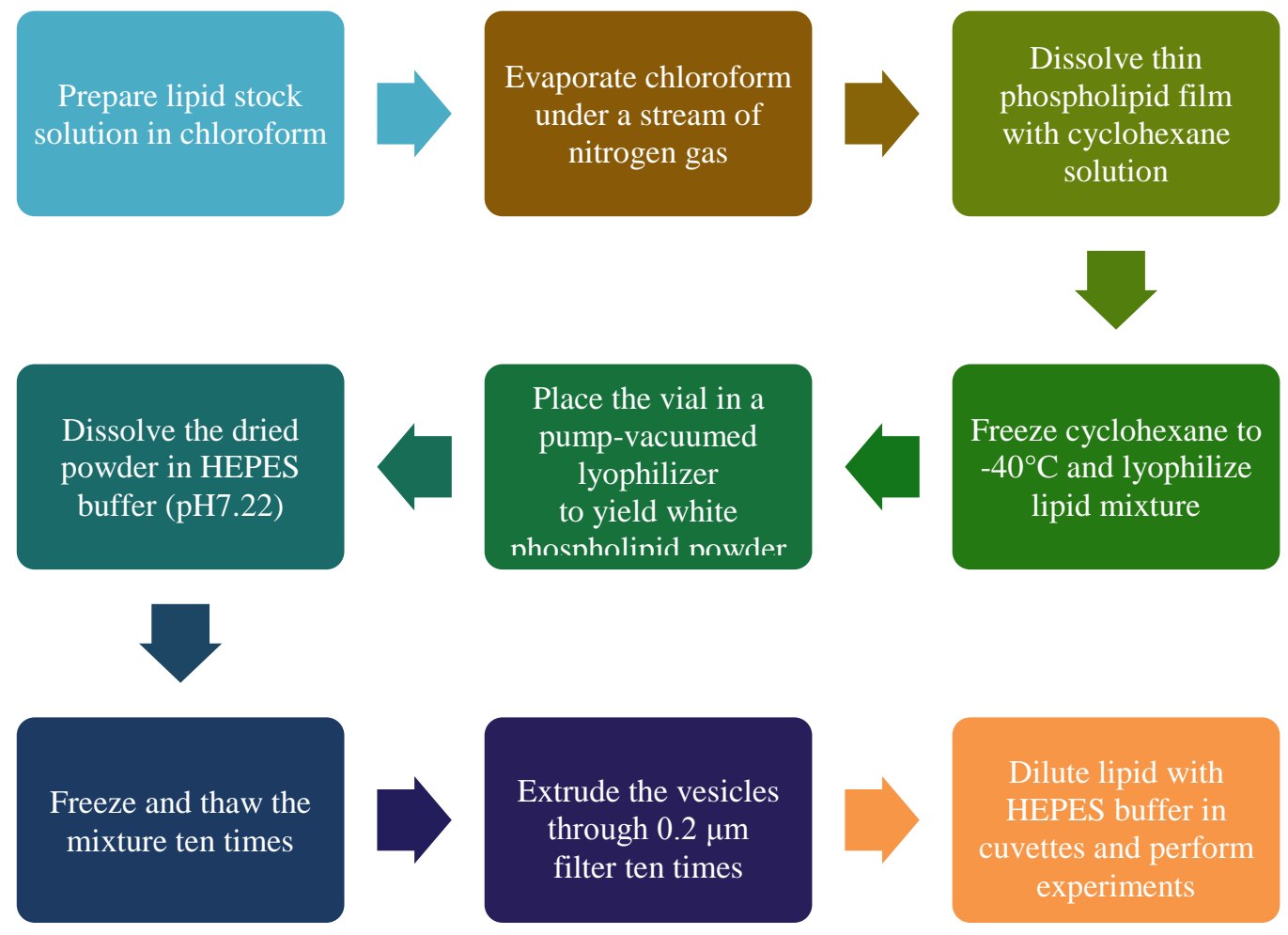

Figure 3.1: Flow Chart of Production of LUVs 


\section{Methods}

\subsection{Background: Jablonski Energy Diagram}

Jablonski diagrams describe the electronic states of molecules and the transitions when electromagnetic radiation is absorbed and emitted. A typical Jablonski diagram is used to illustrate the physics of fluorescence. The definition of fluorescence is the emission of light from atoms or molecules that absorb the energy from radiation. The absorbed energy excites the atoms or molecules. Fluorescence is the decay of an excited state back to the ground state by emission of a photon, and it can exist in different electronic states. The ground electronic state is denoted by $\mathrm{S}_{0}$, first and second electronic state are denoted by $\mathrm{S}_{1}$ and $\mathrm{S}_{2}$. Each of these electronic states, or electronic energy levels, has a number of vibrational energy sublevels depicted by $0,1,2$ etc., as shown in Figure 4.1. Typically, absorption occurs from atoms or molecules with the lowest vibrational energy. However, thermal energy is not adequate to significantly populate the excited vibrational states at room temperature. Naturally, the larger energy difference between the $S_{0}$ and $S_{1}$ excited states is generally too large for thermal population of $S_{1}$ this is why we use light instead of heat to induce fluorescence.



Figure 4.1: Jablonski Energy Diagram for Fluorescence [51] 
Following ultraviolet or visible light absorption, many processes can occur. A fluorophore in the ground state is usually excited to a higher vibrational level of the $S_{1}$ or $S_{2}$ from the ground state $S_{0}$. The molecules rapidly relax and back down to the lowest vibrational level of $\mathrm{S}_{1}$ via a non-radiative process. This process is called internal conversion and generally occurs in $10^{-12}$ second or less. Since the lifetimes of fluorescence are very short typically near $10^{-8}$ second, internal conversion is generally complete prior to photon emission. Thus, emission of fluorophores generally results from the lowest vibrational level of $S_{1}$. Then from the lowest excited state $S_{1}$, the molecules decay (relax) back to the ground state by emitting a photon, the process is called fluorescence. Therefore, when fluorophores return to different vibrational levels of $S_{0}$ state results in the emission spectrum. Indeed, the emission wavelength is independent of the absorption wavelength because the electron always decays to the lowest vibrational energy level of the excited state before it emits the photon and moves back to the ground state. Interestingly, when emission happens from the higher vibrational ground state, the emission spectrum is usually a mirror image of the absorption spectrum. This result according to the Franck-Condon principle, all-electronic transitions are vertical, and they occur without change in the position of the nuclei. As a result, according to FranckCondon factor, if a particular transition probability between the $0^{\text {th }}$ and $2^{\text {nd }}$ vibrational levels is largest in absorption, the reciprocal transition is also most probable in emission.

\subsection{Definition of Absorbance and Optical Density}

Although absorbance and optical density both measure the absorption of light when that light passes through an optical component, these two terms are not the same. 
Optical density (OD) is a measurement of a refractive medium or optical component's ability to slow or delay the transmission of light. It measures the speed of light through a substance, affected primarily by the wavelength of a given light wave. The slower that light is able to travel through a given medium, the higher the optical density of the medium, thus, it can illustrate that OD measures the amount of attenuation, or intensity lost, when light passes through an optical component, and it also tracks attenuation based on the scattering of light.

In contrast to optical density, absorbance measures the ability of a refractive medium or optical component to absorb light. This sounds incredibly similar but is not quite the same. Where optical density measures the speed of light passing through a medium, absorbance measures how much light is lost over the course of light's passage through the given medium; absorbance considers only the absorption of light within the optical component, and optical density takes the scattering, or refraction, of light into consideration. However, both optical density and absorbance can be tracked through the use of a spectrophotometer.

\subsection{Ultraviolet-Visible Measurements}

An Agilent 8453 Ultraviolet-Visible spectrophotometer (UV-Vis) with a 1,024element diode array was used for the absorbance measurements to monitor the kinetics of the solubilization of lipid membranes by the polymer SMA 3:1 and 2:1. Deuterium lamp tungsten lamps provided the light source for the UV and the visible and Short-Wave Near-Infrared Spectrometer (SWNIR) wavelength range. These lamps emit light in the ranges of approximately $190-800 \mathrm{~nm}$ and $370-1100 \mathrm{~nm}$, respectively. The kinetics of the 
solubilization of lipid membranes by the polymers was measured at $300 \mathrm{~nm}$ at $25^{\circ} \mathrm{C}$ using a $1 \mathrm{~cm}$ path length quartz cuvette. The absorbance was measured every minute for 30-45 minutes.

\subsection{Fluorescence Spectroscopy}

Fluorescence lifetime and anisotropy measurements were performed with a Frequency-Domain Chronos Lifetime Spectrometer (ISS, Urbana, IL). Diode laser provides excitation at $376 \mathrm{~nm}$ for DPH. Lifetime and anisotropy decay data were acquired using decay acquisition software from ISS at $25^{\circ} \mathrm{C}$. For lifetime and anisotropy measurements, 15 modulation frequencies were used, logarithmically spaced from 2 to $250 \mathrm{MHz}$. All lifetime measurements were made with the emission polarizer at the magic angle of $54.7^{\circ}$ relative to the vertically polarized excitation beam. The reference cuvette for DPH was filled with 1,4-bis(5-phenyl-2-oxazolyl)benzene (POPOP) in ethanol with a reference time of $1.35 \mathrm{~ns}$. The lifetime of the reference was chosen to be comparable with the particular samples for each anisotropy decay measurement, the instrumental polarization factors were measured and found to be between 1 and 1.05 , and the appropriate correction factor was applied. At each frequency, data were accumulated until the standard deviations of the phase and modulation ratio were below 0.2 and 0.004 , respectively, and these values were used as the standard deviation for the measured phase and modulation ratio in all subsequent analysis. The lifetime and anisotropy decay were measured at $25^{\circ} \mathrm{C}$ without and with different amounts of SMA polymer. 


\subsubsection{Light Scattering}

In this study, Rayleigh scattering is observed. This scattering occurs when the particles are smaller than the wavelength of the light being scattered. Therefore, this type of scattering is wavelength dependent. As the wavelength decreases, the amount of scattering increases. The size of a scattering particle is often parameterized by the ratio

$$
x=\frac{2 \pi r}{\lambda}
$$

where $r$ is its characteristic length (radius) and $\lambda$ is the wavelength of the light. The amplitude of light scattered from within any transparent dielectric is proportional to the inverse square of its wavelength and to the volume of material that is to the cube of its characteristic length. The wavelength dependence is characteristic of dipole scattering and the volume dependence will apply to any scattering mechanism. Because the particles are randomly positioned, the scattered light arrives at a particular point with a random collection of phases; it is incoherent and the resulting intensity is the sum of the squares of the amplitudes from each particle and therefore proportional to the inverse fourth power of the wavelength and the sixth power of its size. In detail, the intensity $I$ of light scattered by any one of the small spheres of diameter $d$ and refractive index $n$ from a beam of unpolarized light of wavelength $\lambda$ and intensity $I_{0}$ is given by

$$
I=I_{0} \frac{1+\cos ^{2} \theta}{2 R^{2}}\left(\frac{2 \pi}{\lambda}\right)^{4}\left(\frac{n^{2}-1}{n^{2}+2}\right)^{2}\left(\frac{d}{2}\right)^{6}
$$

where $R$ is the distance to the particle and $\theta$ is the scattering angle. Averaging this over all angles gives the Rayleigh scattering cross-section

$$
\sigma_{\mathrm{s}}=\frac{2 \pi^{5}}{3} \frac{d^{6}}{\lambda^{4}}\left(\frac{n^{2}-1}{n^{2}+2}\right)^{2}
$$


The fraction of light scattered by a group of scattering particles is the number of particles per unit volume $N$ times the cross-section. The strong wavelength dependence of the scattering $\left(\sim \lambda^{-4}\right)$ means that shorter (blue) wavelengths are scattered more strongly than longer (red) wavelengths. In our experiment, the size of LUVs is $200 \mathrm{~nm}$, and the wavelength is $300 \mathrm{~nm}$, therefore, the Rayleigh scattering occurs. In addition, we measure the optical density are used as a measure of the concentration of LUVs in a suspension. As UV/visible light passes through a cell suspension, the light is scattered where greater scatter indicates that more LUVs is present. However, the decrease in scattering or optical density indicates the start formation of nanodiscs by the SMA and disappears of vesicles. 


\section{Frequency-Domain Lifetime and Anisotropy Measurement}

\subsection{Time-resolved Fluorescence (Intensity Decays)}

There are two types of fluorescence measurements, steady state and time-resolved (or fluorescence lifetime) spectroscopies. Steady-state fluorescence measurement does not provide the kinetic information of fluorescent macromolecules during measuring intensity decay or anisotropy decay, thus, time-resolved fluorescence measurement is an important tool in biochemical research [52]. There are two main techniques to obtain lifetime measurements: time (or pulsed) domain and frequency (or harmonic) domain [53]. In time-domain measurements, the sample is excited by a short-pulsed light source, and the intensity decay of fluorescence is measured. Usually the time-resolved emission light intensity is modeled as the sum of exponential decays. For a single exponential decay, the lifetime is defined as the time when the value of intensity is $1 / e$ of its initial value. For multi-exponential decay, the resulting time-dependent emission can be described by a sum of multi-exponential functions

$$
I(t)=\sum_{i} \alpha_{i} e^{-t / \tau_{i}}
$$

where $\alpha_{i}$ are the pre-exponential factors and $\tau_{i}$ are the decay times. The fraction of the intensity due to each multi-exponential component is given by

$$
f_{i}=\frac{\alpha_{i} \tau_{i}}{\sum_{j} \alpha_{j} \tau_{j}}
$$

Note that although usually intensity decays are analyzed in terms of the multi-exponential model, the actual decay may not be exponential.

It is rather difficult to obtain accurate data for multiple exponential decay using time-resolved fluorescence measurements, because multiple decay exponential 
parameters are highly correlated, thus it is hard to identify each of them [54]. Frequencydomain measurements offer the advantage of reducing measurement noise to a predefined value via continuous sampling. However, operating within the time-domain, the signal from the reference is subtracted from the sample signal and the resulting curve has intensity close to the noise level of the measurement.

For frequency-domain fluorescence measurement, the intensity of the light source is modulated at a high frequency, which is comparable to the reciprocal of the lifetime. This is necessary because according to Equations 6 and 7, if $\omega$ is very small the phase delay will become zero and the modulation ratio will approach 1 . This is not practical for frequency domain applications because the measurements depend upon a finite phase delay and a modulation ratio smaller than 1. In Figure 5.1, the blue curve is excitation intensity; the orange curve is emission intensity. The time lag between absorption and emission is described as the phase shift $\Phi_{\omega}$. The intensity decay of the emission light results in demodulation by a factor $m_{\omega}$, where $\omega$ is the modulation frequency.



Figure 5.1 Definition of the Phase Angle and Modulation of Emission [55]

It may seem difficult to observe the phase delays and modulation ratio at high frequency, but the measurement is actually easy using cross-correlation detection. While 
the excitation light is modulated at frequency $F$, the detector is modulated at frequency $F+\delta F$ to avoid harmonics. The difference frequency $\delta F$ is usually about $25 \mathrm{~Hz}$.

Both time domain and frequency domain time-resolved measurements are designed to recover the parameters describing the time-dependent decay of the sample. For a single-exponential decay, lifetime $\tau$ can be calculated from phase shift $\phi_{\omega}$ and modulation ratio $m_{\omega}$ by the following equations

$$
\begin{gathered}
\tan \phi_{\omega}=\omega \tau \\
m_{\omega}=\left(1+\omega^{2} \tau^{2}\right)^{-1 / 2}
\end{gathered}
$$

\subsection{Time-resolved Anisotropy Decays}

Fluorescence polarization phenomenon (fluorescence anisotropy) is defined as the emitted light of fluorophore has unequal intensities along different axes of polarization. In measurements of fluorescence anisotropy, the sample is excited with a pulse of vertically polarized light, and the excitation with polarized light will lead to that the emission polarized as well. The electric vector of the excitation light is oriented parallel to the vertical or z-axis. The emission intensity is observed through a polarizer where the parallel intensity $I_{\|}(\mathrm{t})$ and perpendicular intensity $I_{\perp}(\mathrm{t})$ happened when the emission polarizer is oriented parallel or perpendicular to the direction of the polarized excitation, respectively, as shown in Figure 5.2. These polarized intensity decays are used to calculate the time-dependent anisotropy (time-resolved fluorescence anisotropy, or instantaneous anisotropy) as

$$
r(t)=\frac{I_{\|}(t)-I_{\perp}(t)}{I_{\|}(t)+2 I_{\perp}(t)}
$$


where the denominator is the total intensity $I(t)=I_{\|}(\mathrm{t})+2 I_{\perp}(\mathrm{t})$. If $I_{\|}=3 I_{\perp}$ anisotropy reaches maximum value 0.4 .


Figure 5.2: Frequency-Domain Measurements of Anisotropy Decay [56, 57]

From Equation 8, we notice that for time domain anisotropy decay measurement, the difference between two noisy signals of $I_{\|}$and $I_{\perp}$ in the numerator yields a curve that has two times the noise of the regular signal. However, the difference between two noisy can fall below a preset value if used in frequency-domain. Therefore, the frequency domain measurement is superior to the time domain for anisotropy measurement.

Empirically all anisotropy decays can be described as a sum of exponentials

$$
r(t)=\left(r_{0}-r_{\infty}\right)\left[\beta_{1} e^{-t / \varphi_{1}}+\beta_{2} e^{-t / \varphi_{2}}\right]+r_{\infty}
$$

where $\varphi_{1}$ and $\varphi_{2}$ are the individual correlation times, $r_{0}$ is the fluorescence anisotropy at $t=0$, and $r_{\infty}$ is the non-decaying anisotropy, as shown in Figure 5.3. 


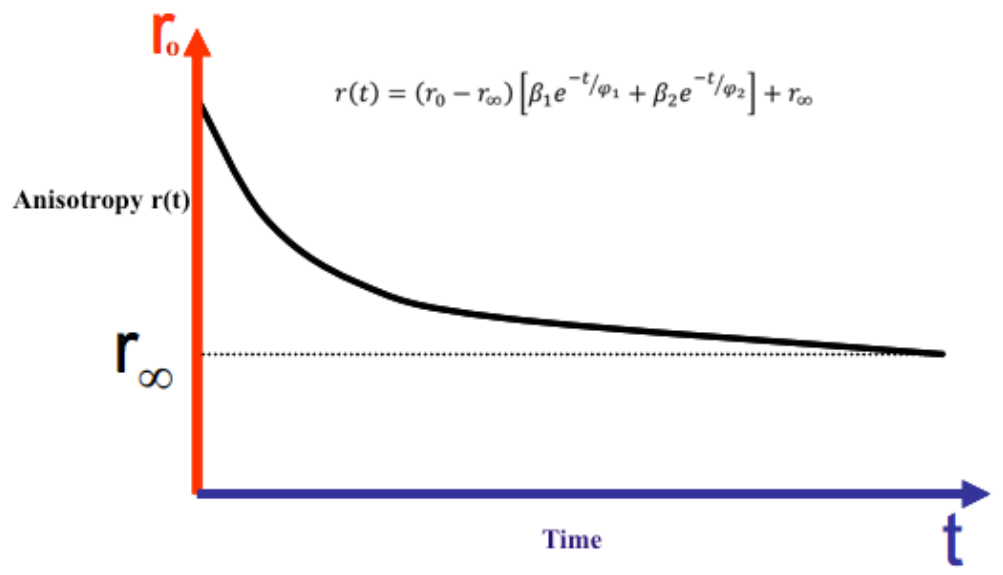

Figure 5.3: Example of Anisotropy Decay [58]

Similar to the frequency domain in lifetime measurement, the frequency domain in anisotropy decay has two parameters characterize the anisotropy decay, (phase angle) phase shift $\Delta_{\omega}$ between the perpendicular and parallel components of the emission light, and the amplitude ratio $\Lambda_{\omega}$ of the parallel and perpendicular components of the modulated emission, where $\omega$ is the modulation frequency

$$
\begin{aligned}
& \Delta_{\omega}=\phi_{\perp}-\phi_{\|} \\
& \Lambda_{\omega}=m_{\|} / m_{\perp}
\end{aligned}
$$

A commonly used model-independent order parameter derived from anisotropy decays is the parameter $S$. The value of square root of $r_{\infty}$ divided by $r_{0}$ is defined as order parameter $S$ [59]

$$
S=\sqrt{\frac{r_{\infty}}{r_{0}}}
$$

The value of order parameter ranges from 0 to 1 . When the value of $r_{\infty}$ is close to $r_{0}$, the value of $S$ is close to 1 ; when $r_{\infty}$ is much smaller than $r_{0}$, then $S$ is close to 0 . 


\subsection{Rotational Diffusion Model}

The empirical sum-of-exponentials model in Equation 10 provides information about fluorophore rotational correlation times, but it does not provide information regarding the range of equilibrium angular orientations of the fluorescent probe that restricted by surrounding phospholipid acyl chains [93]. Therefore, the Brownian rotational diffusion (BRD) model was used to analyze the data in anisotropy decays. The BRD model is based on an approximate solution of the Smoluchowski equation [61, 62], and it yields the order parameters $\left\langle P_{2}\right\rangle$ and $\left\langle P_{4}\right\rangle$ that can be used to construct an orientation distribution function $f(\theta)$ of the probe molecule. Also, the BRD model provides a theoretical framework of the equilibrium orientation distribution of a freetumbling fluorescent probe with cylindrical symmetry which reflects the equilibrium orientation order of the surrounding phospholipid acyl chains. In general, the orientation of a cylindrically symmetric molecule in a lipid bilayer can be described by the angle $\theta$ between the position of its symmetry axis and the local membrane normal. The orientation distribution function $f(\theta)$ generally written as a series expansion of the Legendre polynomials $P_{n}(\cos \theta)$

$$
f(\theta)=\sum_{n} \frac{1}{2}(2 n+1)\left\langle P_{n}\right\rangle P_{n}(\cos \theta)
$$

where $n$ is even and $\left\langle P_{n}\right\rangle$ is the $n$th rank orientation order parameter. $\left\langle P_{n}\right\rangle$ are calculated as

$$
\left\langle P_{n}\right\rangle=\int_{0}^{\pi}(\sin \theta) f(\theta) P_{n}(\cos \theta) d \theta
$$

For macroscopically isotropic systems measurements, only the first two order parameter $\left\langle P_{2}\right\rangle$ and $\left\langle P_{4}\right\rangle$ can be extracted from the experimental data. The BRD model relates the observed anisotropy decay with the order parameters $\left\langle P_{2}\right\rangle$ and $\left\langle P_{4}\right\rangle$, the diffusion 
coefficient for rotation about the symmetry axis of the molecule $D_{\perp}$, and $r_{0}$, according to van der Meer et al [60] by

$$
r(t)=r_{0}\left(\sum_{i=1}^{3} g_{i} \exp \left(-t / \varphi_{i}\right)+g_{4}\right)
$$

where

$$
\begin{gathered}
g_{1}=\frac{1}{5}+\frac{2}{7}\left\langle P_{2}\right\rangle+\frac{18}{35}\left\langle P_{4}\right\rangle-\left\langle P_{2}\right\rangle^{2} \\
g_{2}=\frac{2}{5}+\frac{2}{7}\left\langle P_{2}\right\rangle-\frac{24}{35}\left\langle P_{4}\right\rangle \\
g_{3}=\frac{2}{5}-\frac{4}{7}\left\langle P_{2}\right\rangle+\frac{6}{35}\left\langle P_{4}\right\rangle \\
g_{4}=\left\langle P_{2}\right\rangle^{2} \\
\varphi_{1}=g_{1} /\left[6 D_{\perp}\left(\frac{1}{5}+\frac{\left\langle P_{2}\right\rangle}{7}-\frac{12}{35}\left\langle P_{4}\right\rangle\right)\right] \\
\varphi_{2}=g_{2} /\left[12 D_{\perp}\left(\frac{1}{5}+\frac{\left\langle P_{2}\right\rangle}{14}+\frac{8}{35}\left\langle P_{4}\right\rangle\right)\right] \\
\varphi_{3}=g_{3} /\left[12 D_{\perp}\left(\frac{1}{5}-\frac{\left\langle P_{2}\right\rangle}{7}-\frac{2}{35}\left\langle P_{4}\right\rangle\right)\right]
\end{gathered}
$$

The value of $\left\langle P_{2}\right\rangle,\left\langle P_{4}\right\rangle, D_{\perp}$, and $r_{0}$ can be obtained using the least square analysis. However, the resulting series of $\left\langle P_{2}\right\rangle$ and $\left\langle P_{4}\right\rangle$ can produce negative values of $f(\theta)$ using Equation 14. Therefore, it has to be considered that the orientational probability function has to be positive and the total orientational probability has to be 1 , the values of $\left\langle P_{2}\right\rangle$ and $\left\langle P_{4}\right\rangle$ must satisfy additional constraints so that

$$
\begin{gathered}
f(\theta) \geq 0 \\
\int_{0}^{\pi} f(\theta) \sin \theta d \theta=1
\end{gathered}
$$


The results of the analysis with the BRD model based on the angular distribution function $f(\theta)$ that is symmetrical about $\theta=\pi / 2$, and on maximizing the information entropy of $f(\theta)$ $[63,64]$

$$
f(\theta)=N^{-1} \exp \left[\lambda_{2} P_{2}(\cos \theta)+\lambda_{4} P_{4}(\cos \theta)\right]
$$

where $\lambda_{2}$ and $\lambda_{4}$ are constants determined by simultaneous solution of Equation 15 for $\left\langle\left\langle P_{2}\right\rangle\right.$ and $\left\langle P_{4}\right\rangle$, and $N$ is the normalization constant determined by Equation $16 \mathrm{~b}$.

It is useful to calculate a single parameter that is corresponding to the equilibrium orientational freedom restricted by the phospholipid acyl chains. Thus, a comparison of $f(\theta)$ and an isotropic, random distribution was formulated.

$$
f_{\text {random }}=1-\frac{1}{2} \int_{0}^{\pi}\left|f(\theta)-f(\theta)_{\text {rand }}\right| \sin \theta d \theta
$$

where $f(\theta)_{\text {rand }}$ is given by Equation 18 , with $\lambda_{2}=\lambda_{4}=0 . f_{\text {random }}$ results from a direct comparison of a random orientational distribution and the entire angular range from 0 to $\pi$, and it provides information regarding the orientational freedom of $D P H^{v i}$, thus $f_{\text {random }}$ is a disorder parameter.

\subsection{Least-Squares Analysis of Frequency-Domain Intensity Decays}

The nonlinear least squares method is usually used to analyze data of frequencydomain. The phase and modulation values can be calculated using sine and cosine transform of the intensity decay $I(t)$

$$
\begin{aligned}
N_{\omega} & =\frac{\int_{0}^{\infty} I(t) \sin \omega t d t}{\int_{0}^{\infty} I(t) d t} \\
D_{\omega} & =\frac{\int_{0}^{\infty} I(t) \cos \omega t d t}{\int_{0}^{\infty} I(t) d t}
\end{aligned}
$$

where $\omega$ is the circular modulation frequency $(2 \pi$ times the modulation frequency in $\mathrm{Hz})$. 
For a sum of exponentials the transforms are

$$
\begin{gathered}
N_{\omega}=\sum_{i} \frac{\alpha_{i} \omega \tau_{i}^{2}}{\left(1+\omega^{2} \tau_{i}^{2}\right)} / \sum_{i} \alpha_{i} \tau_{i} \\
D_{\omega}=\sum_{i} \frac{\alpha_{i} \tau_{i}}{\left(1+\omega^{2} \tau_{i}^{2}\right)} / \sum_{i} \alpha_{i} \tau_{i}
\end{gathered}
$$

The value of phase shift $\phi_{c \omega}$ and demodulation $m_{c \omega}$ can be calculated from the values of

$$
\begin{gathered}
\tan \phi_{c \omega}=\frac{N_{\omega}}{D_{\omega}} \\
m_{c \omega}=\left(N_{\omega}^{2}+D_{\omega}^{2}\right)^{1 / 2}
\end{gathered}
$$

$N_{\omega}$ and $D_{\omega}$ by the following equations

In the least-squares analysis, the parameters $\alpha_{i}$ and $\tau_{i}$ are varied to minimize the value of the goodness-of-fit parameter

where $v$ is the number of degrees of freedom, given by the number of data points

$$
\chi_{R}^{2}=\frac{1}{v} \sum_{\omega}\left[\frac{\phi_{\omega}-\phi_{c \omega}}{\delta_{\phi}}\right]^{2}+\frac{1}{v} \sum_{\omega}\left[\frac{m_{\omega}-m_{c \omega}}{\delta_{m}}\right]^{2}
$$

subtracted by the number of parameters. $\phi_{\omega}$ and $m_{\omega}$ are measured data of phase and modulation, $\delta_{\phi}$ and $\delta_{m}$ are the standard deviation of the phase and modulation values. 


\section{Instrumentation}

\subsection{Agilent 8453 UV-Visible Spectrophotometer}

The Agilent 8453 UV-Visible spectrophotometer (UV-Vis) with a 1,024-element diode array was used for the absorbance measurements. The radiation source for the spectrophotometer is a combination of a deuterium-discharge lamp for the ultraviolet (UV) wavelength equipped with a shine-through an aperture and a tungsten lamp for the visible and shortwave near-infrared wavelength range [65]. The shine-through deuterium lamp is designed based on the existence of the UV and visible light where the light from the tungsten lamp is focused through a small discharge aperture of the deuterium lamp [66]. This allows an axial configuration and improved UV-Vis intensity by placing the two sources in series. The light from both sources receives to the source lens, this lens forms a single and collimated beam of light passes through shutter and stray light filter. The shutter is an electromechanical type that opens to allow the light passes through the sample for measurements. During sample measurements, it closes to prevent the light from entering the sample to collect the data that are required; this is because overexposure to light that could affect the data is not being acquired. Another component installed adjacent to the shutter is the stray light correction filter. Stray light comes from different sources, these sources include 1/ Scattering by the grating and mirror surfaces, 2/ scattering by surfaces of lenses and filters, 3/ Rayleigh and Mie scattering by dust particles in the air, (4) distortions because of the thermal gradients in the air inside the optical system, (5) diffraction on the apertures due to the light passing through apertures, and many other sources. The stray light correction filter measures the intensity spectra of the reference or the sample by introducing a filter that blocks $50 \%$ of the light entering 
the detection array with a wavelength of $420 \mathrm{~nm}$. The significance of this wavelength is derived experimentally as the amount of stray light will increase substantially at wavelengths below $400 \mathrm{~nm}$. This issue could be addressed with a standard correction matrix; however, no single, standard UV or visible wavelength sources exist for comparison. Therefore, in the Agilent 8453, with the correction filter, first stray light measured and then stray-light intensity is subtracted from the first spectrum to give a stray-light corrected spectrum. This allows for the acquisition of stray-light corrected spectra. The spectrophotometer also has sample compartment where the cuvettes of the sample or reference can place. The cuvette's temperature can be controlled by a thermoelectric cooling element. The collimated light passing through the sample is then collected by the spectrograph, which its components are the spectrograph lens, the slit, the grating, and the photodiode array. The spectrograph lens focused the collimated light beam, the slit is used to control the width of the incoming light and makes sure that each spectral element are addressing the appropriate photodiode, the grating disperses the light into its respective components $(190-1100 \mathrm{~nm}$ for this instrument) by using a concave grating, then, the grating disperses the light onto the diode array at an angle linear proportional to the wavelength. Figure 6.1 represents the Agilent 8453 UV-visible spectroscopy system. 



Figure 6.1: Optical System Housed in Agilent 8453 UV-Visible Spectrophotometer [67]

\section{1.1 Fluorescence Spectra}

When the light sources pass through a solution, a certain portion of the light is absorbed by the substance, and the light can be measured by the spectrophotometer and quantified by defining the term absorbance (A). Absorbance defined as the amount of light absorbed by a solution and is given by

$$
I=I_{0} 10^{-A}
$$

where $I o$ is the intensity of the light incident before it enters the sample and $I$ is the intensity of the light after is passing through the sample (transmitted). In conventional 
applications, the measured values of $I$ and $I o$ are used to calculate absorbance and Equation 26 can be written as

$$
A=\log \left(\frac{I_{0}}{I}\right)
$$

There is a connection between the absorbance and the concentration. This relationship is expressed by Beer's law, which defined as the amount of light absorbed by the substance is proportional to its concentration in solution as follows

$$
A=\varepsilon c d=\alpha d
$$

where $A$ is the absorbance (no unit), $\varepsilon$ is the molar absorptivity of the medium with a unit $\left(\mathrm{L} \mathrm{mol}^{-1} \mathrm{~cm}^{-1}\right), c$ is the concentration of the solution with a unit $\left(\mathrm{mol} \mathrm{L}^{-1}\right), d$ is the path length of the cuvette that passes through the sample with a unit $(\mathrm{cm})$, and $\alpha$ is the absorption coefficient with a unit $\left(\mathrm{cm}^{-1}\right)$.



Figure 6.2: Absorption of Light Passing through a Sample [68]

The absorbance depends on the wavelength of the light, and an instrument called a spectrophotometer or spectrometer measures the absorption spectrum. When a photon of visible light is absorbed by a chromophore, an electron transitions from ground state to into excited state that higher in energy. The excitation spectrum can be determined by monitoring the fluorescent emission at a maximum intensity wavelength while the 
fluorophore is excited through sequential wavelengths. The chosen emission wavelength is just allowed to pass to the detector, and the intensity of the emitted spectrum is measured as a function of wavelength. In fact, the absorbance and excitation spectra are involved in the same processes. This because the two spectra generally have the same shape, and they are superimposable.

\subsection{ISS Chronos Spectrometer}

Chronos FD (frequency-domain fluorescence lifetime instrument) is a spectrometer to measure fluorescence lifetime and anisotropy decay (ISS, Urbana, IL). The components of Chronos fluorimeter as follow: a light source, sample compartment, detectors, wavelength selection, polarizers, computer, and software, as shown in Figure 6.3.

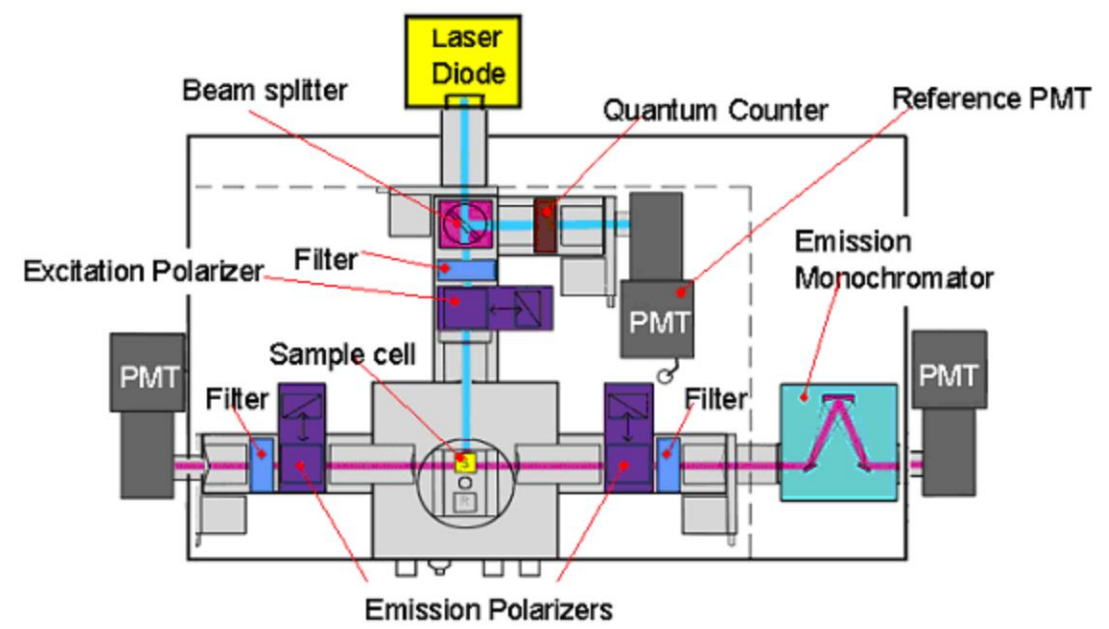

Figure 6.3: Schematic Drawing of ChronosFD, the Frequency-Domain Fluorimeter from ISS [69

The mechanism of operation the frequency domain time-resolved fluorescence system (Chronos) is by the light source (a partially polarized laser diode) is modulated at high frequency by an external function generator outputting a sinusoidal wave with a 
typical frequency range of 5-300 MHz. The frequency of the laser signal which is timevarying is then sent through the excitation polarizer, which is aligned with the laser so the maximum intensity is delivered to the sample cell. The alignment procedure is accomplished via an iterative process of tilting the laser diode assembly until the signal at the non-monochromator PMT becomes highest. However, reference PMT and quantum counter should be used to correct any changes occur such as lasers may vary in intensity with age and the output may have some variance in wavelength. Photons originating from the laser diode are delivered to the reference arm with a 50/50 beam splitter. Predating the use of the photodiode, quantum counters were typically rhodamine-B or any fluorophore that has a constant fluorescent yield over a broad spectral range. However, because of the possible variance in the quality of the fluorophore and the requirement of routine maintenance, the fluorophore quantum counters is replaced in these cases. The photodiode has an even broader spectral range while still offering negligible selfabsorption. Photons that are excited from the lasers are fallen on the reference and then to the sample via stage rotation at each measurement point. The emission photons are collected at $90^{\circ}$ relative to the path of the laser diode and sent through the emission polarizer. This polarizer is very important to distinguish between fluorescent lifetimes and rotational information. In case the polarizer does not exist, we are measuring $I_{\|}(\mathrm{t})+$ $I_{\perp}(\mathrm{t})$. However, this is not the total intensity $I_{\|}(\mathrm{t})+2 I_{\perp}(\mathrm{t})$. Therefore, we need to adjust the emission polarizer oriented under to the so-called "magic angle" for which $I_{\perp}$ would contribute twice as much as $I_{\|}$in the passing light. 

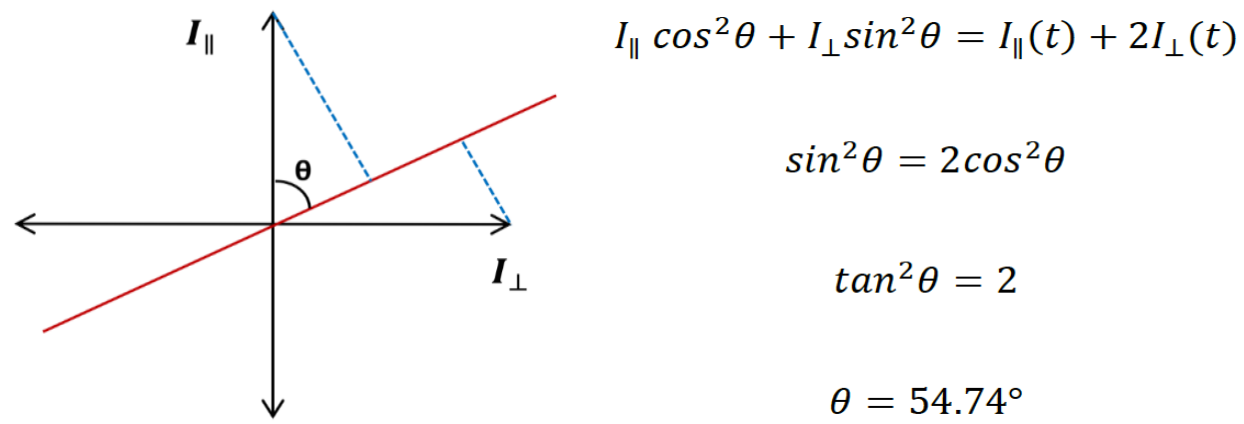

Figure 6.4: Magic Angle

When the emission polarizer is adjusted to the magic angle relative to the polarization of the excitation light, the intensity seen at the PMT represents the total intensity. Therefore, the "magic angle" is conducive for measurements of fluorescent lifetimes. The sample PMT detects the modulated emission from the sample and the reference solution. The lifetime of the reference is known, thus by comparing the phase delay and modulation ratio of the sample and the reference, the lifetime of the sample can be calculated.

However, in case of measurement anisotropy decay, the emission polarizer collect data dynamically rotating between the parallel and perpendicular positions for comparison of sample rotation (i.e. the relative position of the fluorophore). This dynamic anisotropy measurement is accomplished with a constant, vertical excitation polarization. The signals of the emission or excitation can be attenuated by using Neutral density filters, optical elements with constant absorption across a broad spectrum.

Examples of raw data for lifetime and anisotropy measurements are shown in Figures 6.4 and 6.5. 



Figure 6.5 Examples of POPC Lifetime Data with or without SMA $(3: 1)$ at $25^{\circ} \mathrm{C}$
Figure 6.5 displays the lifetime measurement of POPC with DPH at $25^{\circ} \mathrm{C}$ with and without SMA. This figure shows the change in phase delay (blue symbols, left axis) and modulation ratio (green symbols, right axis) with increasing modulation frequency due to the excited state lifetime of the fluorophore. Figure 6.5a, shows the lifetime measurement of the sample without SMA $3: 1$ at $25^{\circ} \mathrm{C}$, by noticing that when the modulation frequency increases, the phase delay increases from approximately (7) to (90), while the modulation ratio decreases from approximately (1) to (0). Figure $6.5 \mathrm{~b}$ shows the lifetime measurement of the sample with SMA $3: 1$ at $25^{\circ} \mathrm{C}$. Figure 6.5c shows the data without SMA 2:1 at $25^{\circ} \mathrm{C}$, and Figure $6.5 \mathrm{~d}$ shows the data with SMA2:1. However, the position of cross points in the figures indicates that 
when the cross points close to the right side that means the sample has a long lifetime and vice versa.


Figure 6.6 Examples of Anisotropy POPC Data

with or without SMA (3:1) at $25^{\circ} \mathrm{C}$
For anisotropy decay measurement at $25^{\circ} \mathrm{C}$, Figure 6.6 shows the change in phase delay and amplitude ratio due to the difference between vertically and horizontally polarized excitation for vertically polarized emission. In the case of anisotropy decay, the changes in phase delay and amplitude ratio is small compared to lifetime measurement. However, it should be noticeable the change of the shape in the two phase-mod graphs. 


\section{Results and Discussion}

\subsection{Kinetics of Membrane Solubilization by SMA}

Many studies [44] have focused on the physico-chemical characterization of SMA-Lipid particles (SMALPs), discoidal particles, or nanodiscs.

The solubilization process can be measured by a decrease in light scattering over time using a spectrophotometer. Lipid vesicles (LUVs) are large particles (hundreds of nanometers up to micrometers in size) and thus efficiently scatter UV light, whereas SMALPs are much smaller and scatter almost no light. Therefore, the solubilization process may be observed by monitoring the decrease in light scattering over time.

The kinetics of vesicle solubilization upon addition of SMA copolymers can be observed by monitoring the decrease in optical density. Initially, the effect was investigated by addition of SMA 2:1 and SMA 3:1 to vesicles of monounsaturated POPC, which undergo a fluid phase transition at $\sim-2^{\circ} \mathrm{C}$.

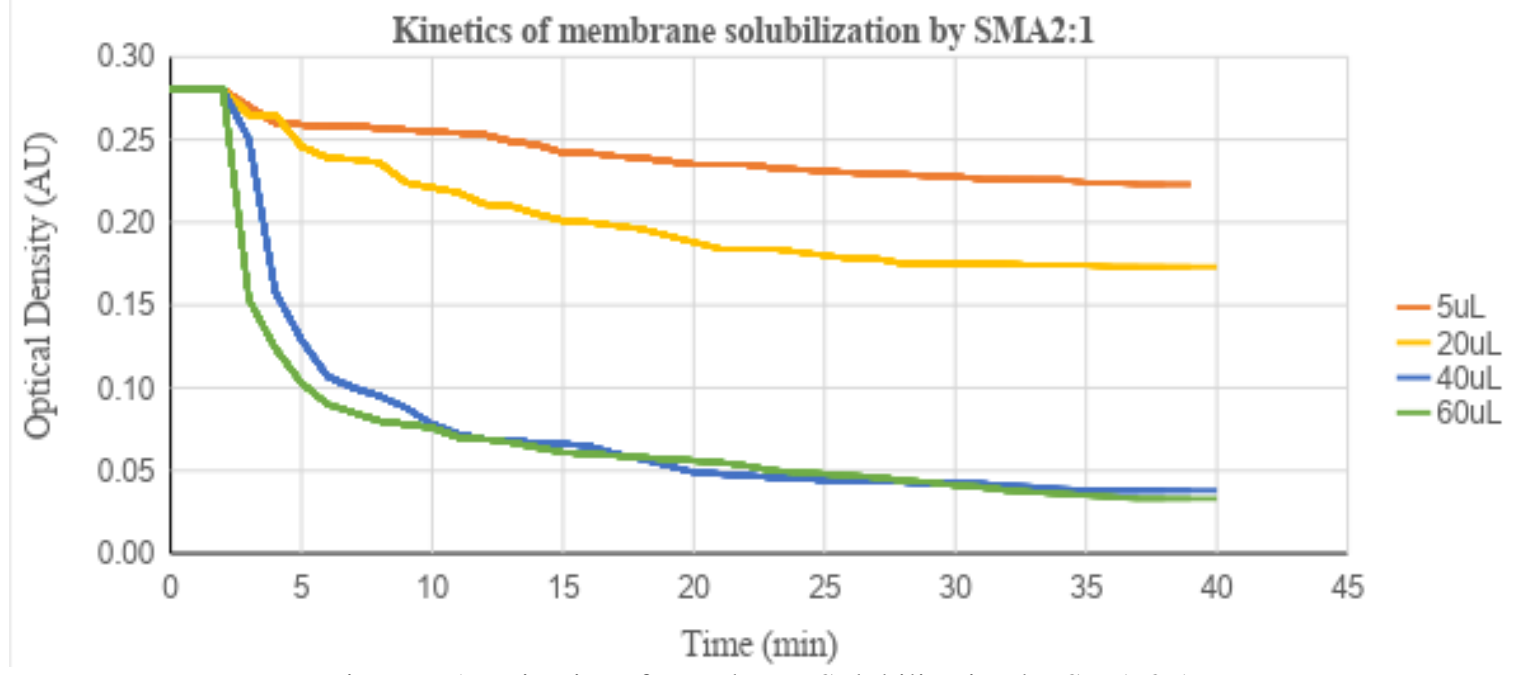

Figure 7.1: Kinetics of Membrane Solubilization by SMA 2:1 
Figure 7.1 shows that the kinetics solubilization of membrane $\mathrm{c}$ of SMA 2:1 at $25^{\circ} \mathrm{C}$. Upon addition of $5 \mu \mathrm{L}$ of $5 \mathrm{mg} / \mathrm{ml}$ polymer to the LUVs, the optical density decreases from $\sim 0.28$ to $\sim 0.23$, which means the SMA starts the solubilization process, and this amount partially solubilizes the LUVs. Increasing the amount of copolymer, decreases the optical density rapidly to $\sim 0.04$, which means the SMA, solubilizes the lipids bilayers almost completely and forms the nanodiscs.

The scattering of light of the sample of POPC is almost stable for the first 3 minutes at wavelength $300 \mathrm{~nm}$; there is more light scattering due to the large size of the vesicles. After adding the SMA 2:1, at 3 minutes the light scattering decreases because the nanodisc (Lipodisq) particles are formed, and they are much smaller than LUVs.



Figure 7.2: Kinetics of Membrane Solubilization by SMA 3:1

Figure 7.2 shows the kinetics of membrane solubilization of SMA $3: 1$ at $25^{\circ} \mathrm{C}$. With the addition of $5 \mu \mathrm{L}$ of $5 \mathrm{mg} / \mathrm{ml}$ polymer to the LUVs, the optical density decreases from $\sim 0.28$ to $\sim 0.24$, which is similar to SMA 2:1 result for the same SMA volume 
added. Increasing the amount of copolymer to 20,40 , and $60 \mu \mathrm{L}$, the optical density decreases quickly to $\sim 0.05$, which means the SMA solubilizes the lipid almost completely and forms the nanodiscs. Both SMAs have the same effect on solubilizing the lipid bilayer.

SMA 2:1 and 3:1 cause fast solubilization above the phase transition temperature of POPC (in fluid phase), and this agrees with other studies done by Dorr, Pardo [45]and Scheidelaar [44], which state that SMA shows a strong preference towards solubilizing the fluid phase. The curve $s$ in figs 7.1 and 7.2 are not smooth, indicating more complex kinetics occurring possibly due to differences in the kinetics of formation and size of intermediate vesicular structures.

The exact formation process of SMALPs is not entirely clear. It has been suggested that there is a three-step model $[41,44]$ where SMAs (1) binds the membrane surface and (2) destabilizes the bilayer before (3) the formation of the nanodiscs.

\subsection{Fluorescence Lifetime}

Figure 7.3 shows the fluorescence lifetime of DPH of SMA 2:1 and SMA3:1. The lifetime of the fluorophore increased from $8.2 \mathrm{~ns}$ to $9.02 \mathrm{~ns}$ and from $8.2 \mathrm{~ns}$ to $~ 9.06$ ns respectively, with increased concentration of SMA 2:1 and SMA 3:1 in the lipid bilayer. Increasing the concentration of copolymers clearly affects the water penetration of the lipid bilayer, which means that the water is more excluded from the lipid bilayer. This implies that thermal motion of phospholipid head groups are will be reduced because SMAs starting to wrap around the hydrophobic core of the bilayer and the lipid packing is tighter. The fact that there is no significant change in the lifetime of the 
fluorophore between the copolymers means the effect on head group packing with respect to water penetration is almost equal.

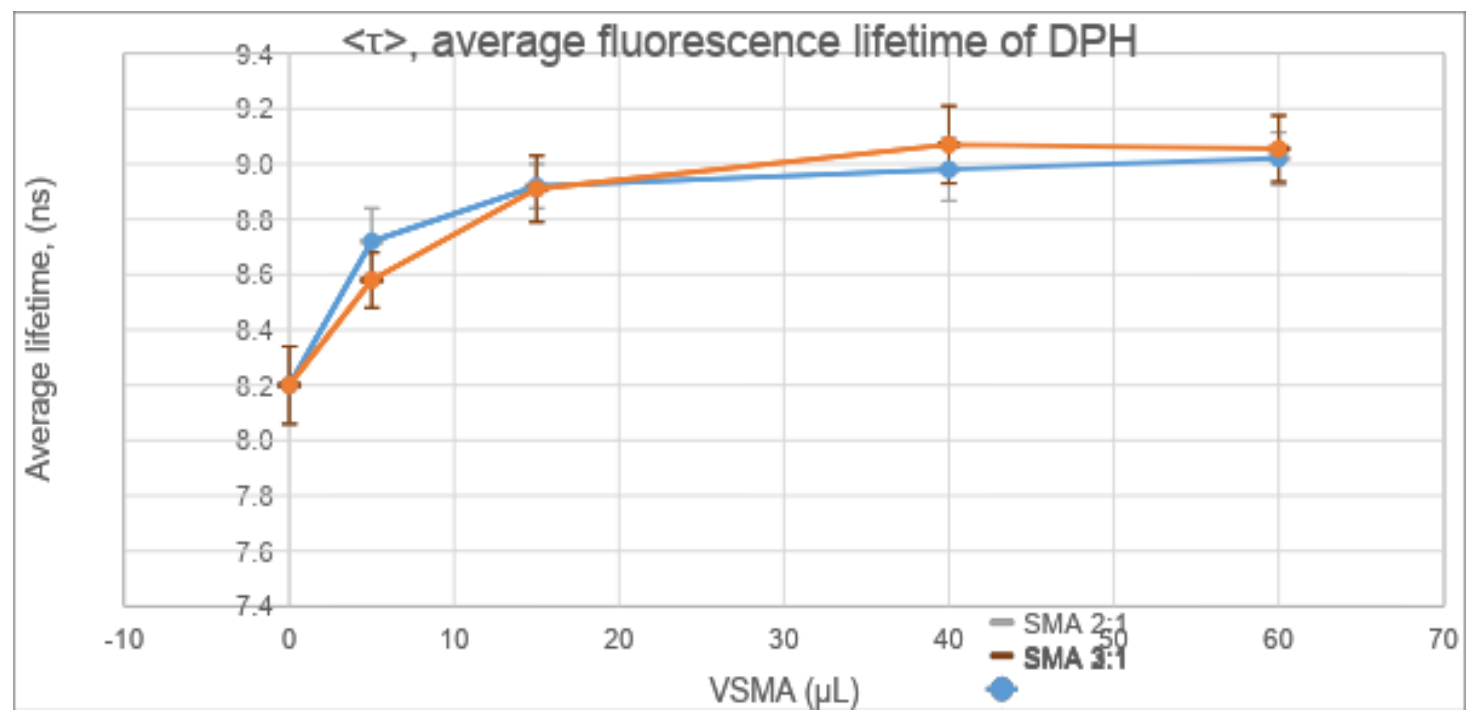

Figure 7.3: Lifetime Change of DPH with SMA 2:1 and SMA 3:1

The variation of the lifetime of DPH is most likely due to changes in water penetration inside the lipid bilayer induced by the change in concentration of the copolymers. Figure 7.3 shows additional of SMA 2:1 and 3:1 both increase the DPH lifetime.

In fact, a study [70] indicates that when membrane dynamics are slow, such as in the gel phase, the distribution of the lifetime values of DPH is relatively broad, and its average value (the center of the distribution) is shifted toward long lifetime values. In the fluid state, the lifetime distribution is relatively narrow and its average value is shifted to shorter lifetime values. The SMA 2:1 and SMA 3:1 results are similar to the gel phase shifts in DPH lifetime indicating that SMA 2:1 and 3:1 alter the head-group or acyl chain packing in the fluid phase. 
Gratton and Parasassi [70] suggested that DPH aligns mainly along the lipid chains and resides in the membrane interior. It has the freedom to diffuse laterally in the membrane, but also to move rapidly along the membrane normal.

\subsection{Dynamic Fluorescence Anisotropy}

The principle of anisotropy measurement has been introduced in Chapter 5.3 and 5.4. From the anisotropy measurement, there are four important parameters derived: rotational correlation time $\langle\phi\rangle$, order parameter $S$, orientational freedom parameter $f_{\text {random }}$, and rotational diffusion coefficient $D_{\perp}$.

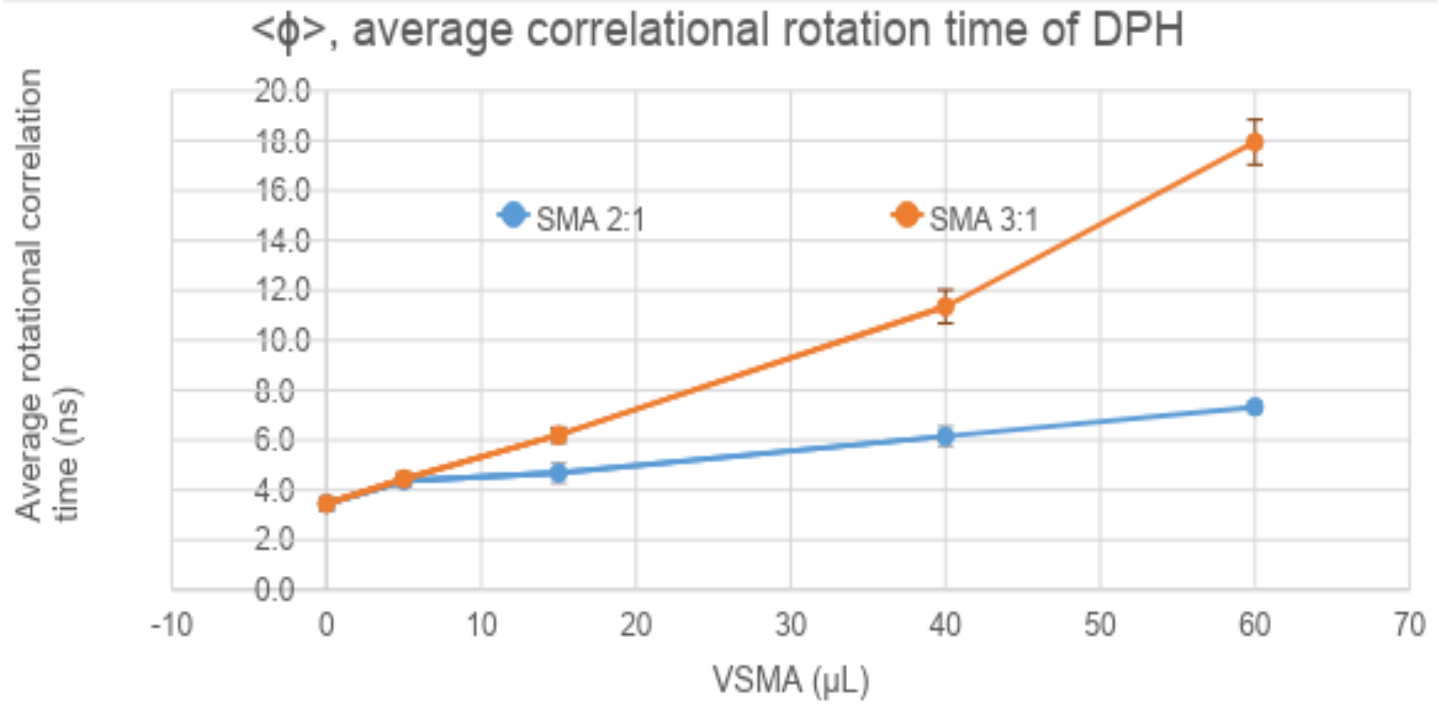

Figure 7.4: Rotational Correlation Time

Figure 7.4 shows the average rotational correlation time $\langle\phi>$ of the DPH with SMA $2: 1,\langle\phi>$ is obtained from model of sum of discrete exponential terms describing rotation (undefined rotation) or average of spin uncorrelated to any axis (the molecule time to spin the dipole of the electric field transition). The average rotational correlation time increases from $\sim 3.45 \mathrm{~ns}$ to $\sim 7.33 \mathrm{~ns}$ with increased concentration of SMA 2:1, which 
means that DPH tumbles slower with more SMA present in the lipid solution. This implies that the acyl chains become close packed and limit the ability of DPH to move. The parameter $\langle\phi\rangle$ is a motional parameter so does not directly report changes in lipid order, but an increase in its value suggests increased order of the lipid that restricts the motion of the lipids chains.

Figure 7.4 also shows the average rotational correlation time $\langle\phi\rangle$ of DPH with SMA 3:1. The rotational correlation time increases from $\sim 3.45 \mathrm{~ns}$ to $\sim 17.49 \mathrm{~ns}$ with increased concentration of SMA 3:1. This huge increase means that the acyl chains stick together tightly and DPH has less ability to move; increased lipid acyl chain order is correlated to reduced motion of the chains. By comparing the results of both SMAs, SMA 3:1 has more impact on the order of the lipid bilayer, which means it is not good as SMA 2:1 for nanodisc extraction because SMA 3:1 changes the environment of the lipids bilayer more. SMA 2:1 results in nanodiscs that are more similar to native LUVs [45], and this is more useful for studying membrane-bound proteins.

Figure 7.4 shows SMA 3:1 increases rotational correlation time, meaning DPH tumbles more slowly with more SMA 3:1 present. We see that SMA 3:1 has a stronger effect on rotational correlation time at higher amounts of SMA 3:1. 


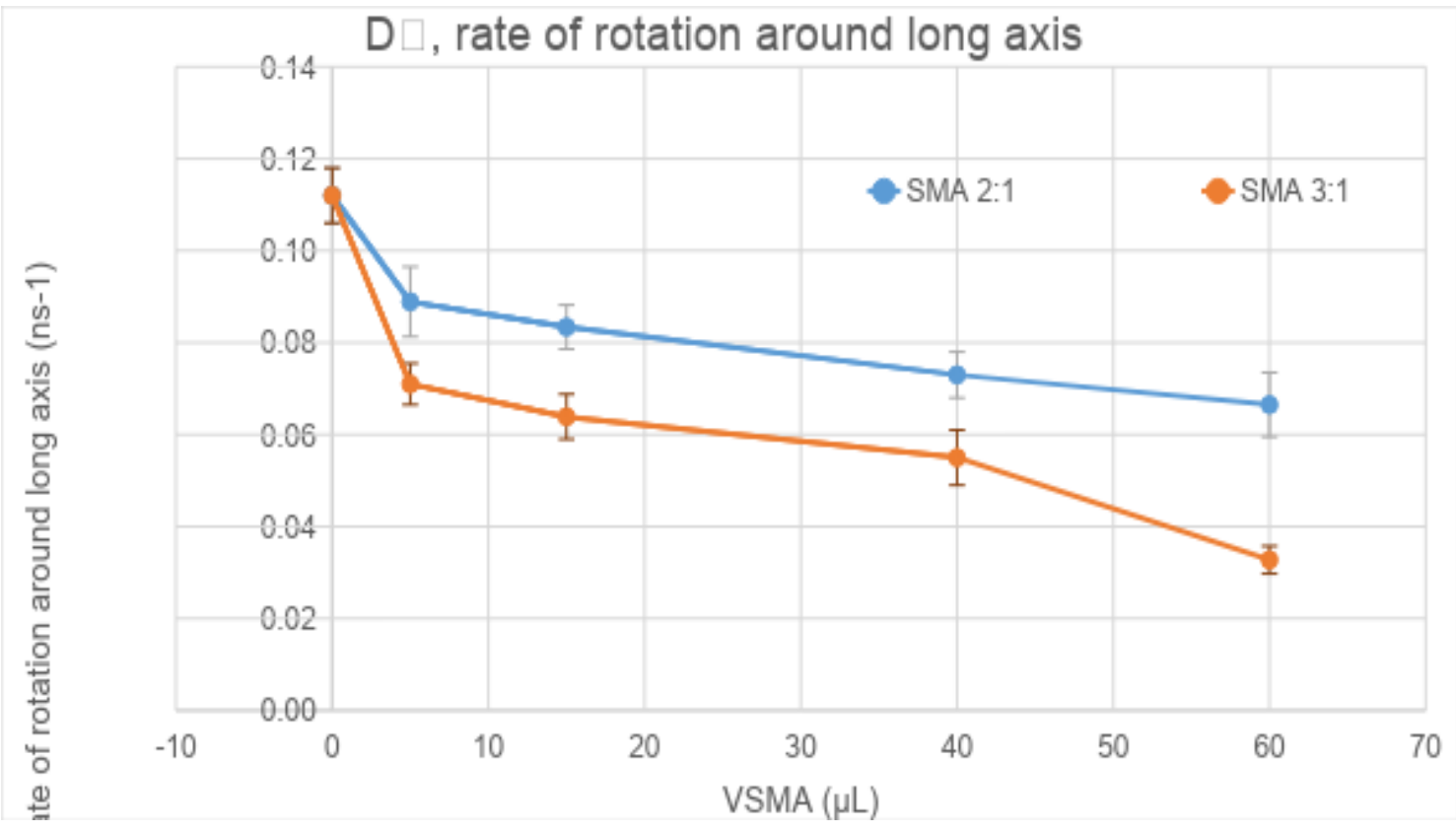

Figure 7.5: Rotational Diffusion Coefficient

Figure 7.5 show the rate constant of rotation around the long axis (motion) $D_{\perp}$. These figures shows SMA decreases rotational diffusion coefficient, which means that DPH rotates slower along its longest axis when more SMA is present. Increased SMA 2:1 slows DPH by a factor of two, SMA 3:1 slows DPH by factor of four which means SMA 3:1 has more impact on the motion of DPH more than SMA 2:1. 




Figure 7.6: Order Parameter $S$

Figure 7.6 shows that SMA 2:1 and 3:1 increase order parameter $S$ from $\sim 0.246$ to $\sim 0.31$ and from $\sim 0,264$ to $\sim 0.345$ respectively, which means SMA 3:1 has more impact on the order of DPH in the lipid bilayer. This parameter is from the model sum-ofexponentials analysis of the anisotropy decay data Equation 9. It is obvious from the figure that SMA 3:1 has much larger effect than 2:1, and both polymers may be reaching a plateau, large error bars make it hard to tell if a further addition of either polymer would lead to further increases in $S$. 


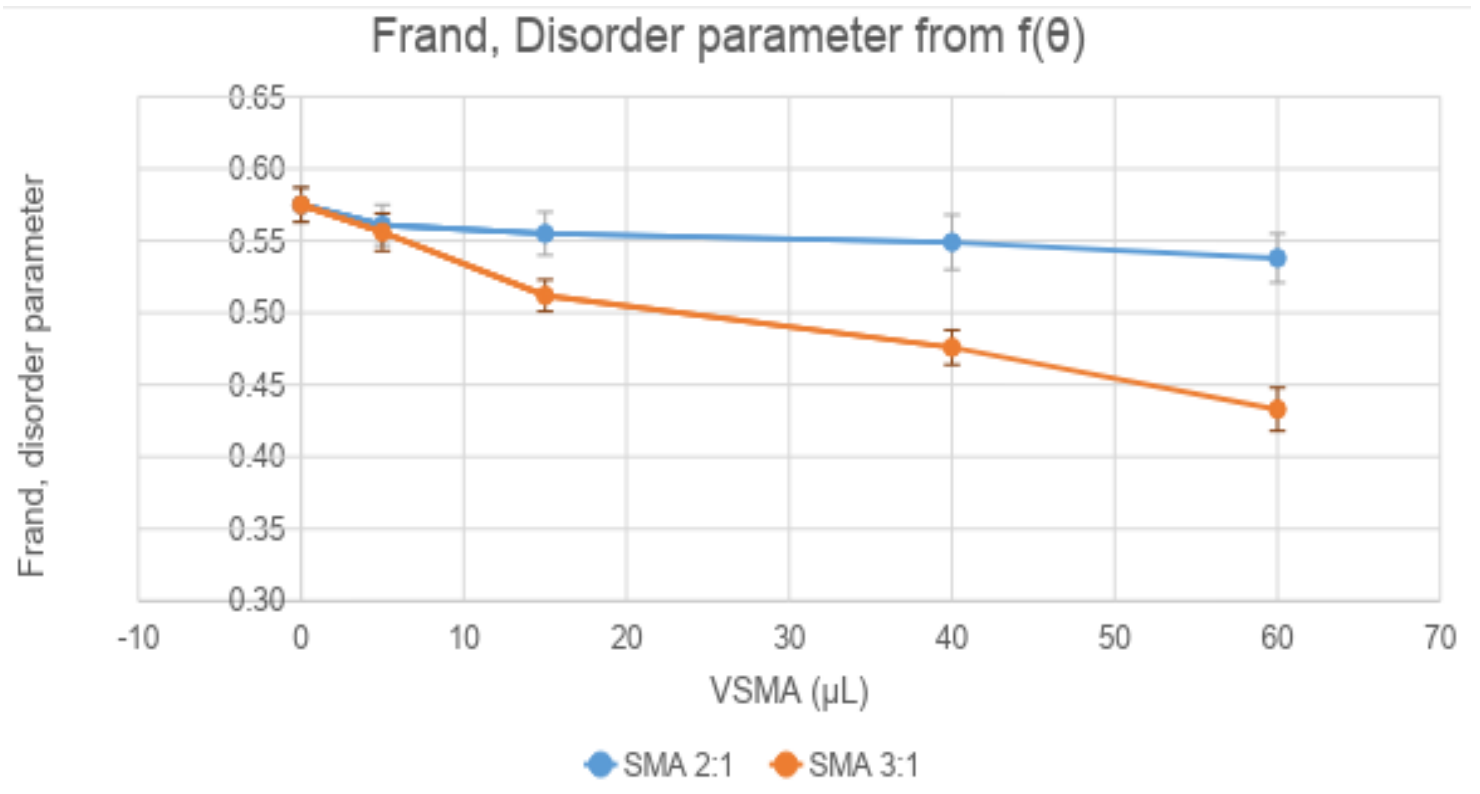

Figure 7.7: Orientational Freedom Parameter $f_{\text {random }}$ (Disorder Parameter $f_{\text {random}}$ )

Figure 7.7 shows the disorder parameter $\boldsymbol{f}_{\text {random }}$ changing with increased SMA 2:1 and SMA 3:1. When the concentration of SMA 2:1 increases, the orientational freedom parameter decreases from $\sim 0.57$ to $\sim 0.53$, and decreases from $\sim 0.57$ to $\sim 0.43$ for SMA 3:1. As introduced in Chapter 5.4, $\boldsymbol{f}_{\text {random }}$ is the overlap of the orientational distribution and a random orientational distribution. We see that at $25^{\circ} \mathrm{C} \mathrm{DPH}$ loses more orientational freedom with increasing amount of SMA 3:1 than SMA 2:1. This means $\boldsymbol{f}_{\text {random }}$ increases the ability of the lipid bilayer to restrict the equilibrium angular orientation of DPH and the low values of SMA 3:1 demonstrate the orientational distributions in a lipid bilayer are restricted with increased concentration of SMA 3:1 than 2:1. (When lipid bilayer more ordered this means the DPH cannot move and distribute between the bilayer, more packed bilayers). 




Figure 7.8: Orientation Distribution Functions

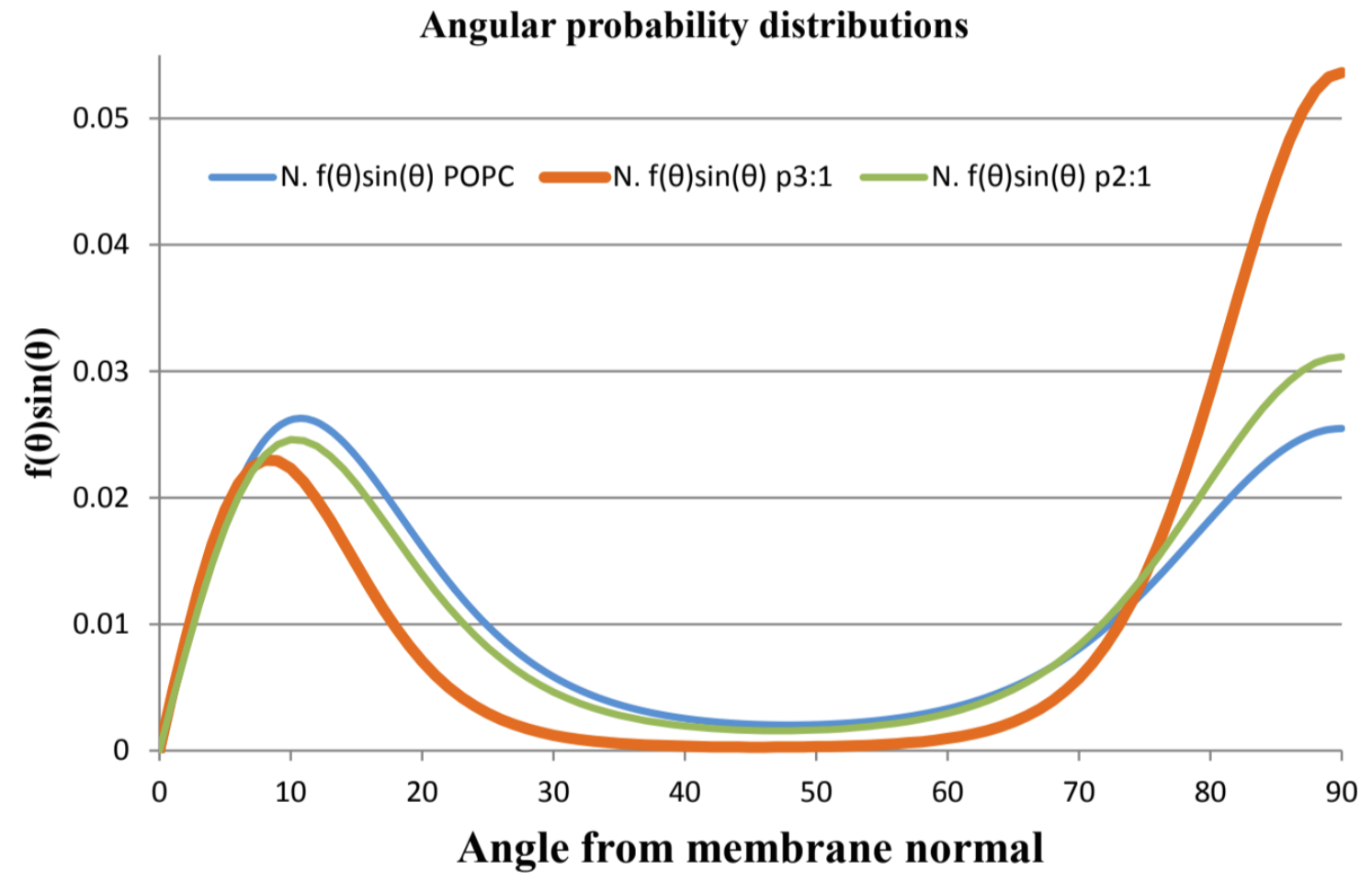

Figure 7.9: Orientational Probability Distributions

Figure 7.8 shows the orientation distribution function $f(\theta)$ for POPC. The distribution functions, $f(\theta)$, are normalized according to Equation 16b; thus the higher the 
value of $f(\theta)$ at $\theta=0$, the greater area under the curve. The effect of increasing concentration of both SMAs is a redistribution from orientations about the bilayer normal to orientations approximately parallel to the plane of the bilayer. The probability density centered at $90^{\circ}$ from the bilayer normal is interpreted as corresponding to the presence of DPH in the bilayer midplane, between the two-monolayer leaflets.

Figure 7.9 shows the orientational probability distributions for DPH with no polymer present and with $60 \mu \mathrm{L}$ of the two polymers present. Here we will answer the question, how does the environment of the phospholipid bilayer alter the orientational distribution of the probe molecule from a random distribution? Figure 7.9 shows the range of changes in the DPH orientational populations induced by increased concentration of SMAs. The blue and green curves show the 2:1 polymer has only a small effect on the equilibrium DPH orientation. They both show about an even split between the population associated with the bilayer normal and population at $90^{\circ}$, which is assumed to be in the bilayer midplane. The orange curve shows that the 3:1 polymer dramatically reduces the bilayer normal population and increases the amount of DPH in the bilayer midplane. 


\section{Conclusions}

Copolymers of styrene and maleic acid (SMA) have gained great attention as alternatives to conventional detergents, as they offer decisive advantages for studying membrane proteins and lipids. The data shows that these two SMAs rapidly solubilize POPC in the fluid phase. The effects of polymer composition on membrane solubilization show that Lipodisq 2:1 and Lipodisq 3:1 form nanodiscs equally rapidly at similar rates and with similar concentration dependence by monitoring the drop in optical density when LUVs solubilize. In addition, lifetime measurements show that both polymers increase the DPH fluorescence lifetime similarly. These results indicate that raising the lifetime of the fluorophore with increased concentration of both SMAs affect the water penetration into the lipid bilayer and reduce the thermal motion of phospholipid head groups. Moreover, the anisotropy measurements indicated that both polymers reduce the rate of rotation $\left(\mathrm{D}^{\perp}\right)$ of DPH in the bilayer; SMA 3:1 has a stronger effect than SMA 2:1. Also, the orientational distribution analysis showed that SMA 2:1 had a small effect on the orientation of DPH whereas SMA 3:1 had a major effect on the orientation of DPH.

Overall, the results suggest that the SMA 2:1 polymer is able to preserve nativelike lipid packing properties in the nanodiscs to a higher extent than the SMA 3:1 polymer, which means SMA 2:1 does not affect the order of lipid packing as much, while SMA 3:1 alters the orders acyl chain packing to a greater degree. We can state that SMA 3:1 tightens the lipid bilayer acyl chain packing in a unique way according to the orientational distribution analysis. These results provide evidence that SMA 2:1 is more suitable as a tool for studies where the goal is to study membrane protein function in nanodiscs. 


\section{References}

[1] Gennis, Robert B. Biomembranes: Molecular Structure and Function. New York: Springer-Verlag, 1989.

[2] Alberts, B., Johnson, A., Lewis,J., Raff, M., Roberts, K., and Walter, P. Molecular Biology of the Cell, 4th Edition, NCBI, 2002.

[3] http://en.wikipedia.org/wiki/File:Cell_membrane_detailed_diagram_4.svg

[4] Lombard, Jonathan. "Once upon a time the cell membranes: 175 years of cell boundary research" Biology Direct 9.32(2014)

[5] Bagatolli, Luis A. Jhon H. Ipsen, Adam C. Simonsen, and Ole G. Mouritsen. "An Outlook on Organization of Lipids in Membranes: Searching for a Realistic Connection with the Organization of Biological Membranes" Progress in Lipid Research 49.4 (2010): 378-89.

[6] Danielli, James Frederic, and Hugh Davson. "A Contribution to the Theory of Permeability of Thin Films." Journal of Cellular and Comparative Physiology 5.4 (1935): 495-508.

[7] Robertson, J. D. "New Observations on the Ultrastructure of the Membranes of Frog Peripheral Nerve Fibers." The Journal of Cell Biology 3.6 (1957): 1043-048.

[8] Bangham, A.D., and R.W. Horne. "Negative Staining of Phospholipids and Their Structural Modification by Surface-active Agents as Observed in the Electron Microscope." Journal of Molecular Biology 8.5 (1964): 660-IN10.

[9] Benson, A. A. "On the Orientation of Lipids in Chloroplast and Cell Membranes." Journal of the American Oil Chemists Society 43.5 (1966): 265-70.

[10] Green, D. E. "Membranes as Expressions of Repeating Units." Proceedings of the National Academy of Sciences 55.5 (1966): 1295-1302.

[11] Stoeckenius, W. "Current Models For The Structure Of Biological Membranes." The Journal of Cell Biology 42.3 (1969): 613-46.

[12] Singer, S. J., and G. L. Nicolson. "The Fluid Mosaic Model of the Structure of Cell Membranes." Science 175.4023 (1972): 720-31.

[13] Wang. H., and Li. G. "Membrane Biophysics: new Insights and Methods" Springer.

[14] Shimshick, Edward J., and Harden M. Mcconnell. "Lateral Phase Separation in Phospholipid Membranes." Biochemistry 12.12 (1973): 2351-360.

[15] Marčelja, S. "Lipid-mediated Protein Interaction in Membranes." Biochimica Et Biophysica Acta (BBA) - Biomembranes 455.1 (1976): 1-7.

[16] Chapman, Dennis, and Donald Francis Hoelzl Wallach. "Physical Basis for Trigger Processes and Membrane Structures." Biological Membranes. London: Academic, 1984. 105-43.

[17] Simons, Kai, and Gerrit Van Meer. "Lipid Sorting in Epithelial Cells." Biochemistry 27.17 (1988): 6197-202. 
[18] Simons, K., and E. Ikonen. "Functional Rafts in Cell Membranes." Nature 387 (1997): 569-72.

[19] Simons, K., and R. Ehehalt. "Cholesterol, lipid rafts, and disease" J Clin Invest 110 (2002): 597-603.

[20] Radeva, G., and F. J. Sharom. "Isolation and characterization of lipid rafts with different properties from RBL-2H3 (rat basophilic leukemia) cells" Biochemical Journal 380 (2004): 219-30.

[21] Lonova ,Irina V." Phase Diagram of Ternary Cholesterol/Palmitoylsphingomyelin/Palmitroyloleoyl-Phosphatidylcholine Mixtures: Spin-Label EPR Study of LipidRaft Formation." Biophysical Journal 102 (2012): 1856-865.

[22] De Almeida, Rodrigo F.M., Aleksandre Fedorov, and Manuel Prieto. "Sphingomyelin/Phosphatidylcholine/Cholesterol Phase Diagram: Boundaries and Composition of Lipid Rafts." Biophysical Journal 85.4 (2003): 2406-416.

[23] http://en.wikipedia.org/wiki/File:Lipid_raft_organisation_scheme.svg

[24] Rawicz, W., K.Cc. Olbrich, T. Mcintosh, D. Needham, and E. Evans. "Effect of Chain Length and Unsaturation on Elasticity of Lipid Bilayers." Biophysical Journal 79.1 (2000): 328-39.

[25] https://en.wikipedia.org/wiki/Lipid_bilayer_phase_behavior

[26] Veatch, Sarah L. "Lipids out of Order." Nature Chemical Biology 4.4 (2008): 22526.

[27] Gobley, Theodore N. "Recherches Chimiques Sur Le Jaune D'oeuf - Examen Comparatif Du Jaune D'oeuf Et De La Matière Cérébrale." Journal De Pharmacie Et De Chimie (1847): 409-12.

[28] Campbell, Neal A., Brad Williamson, and Robin J. Heyden. Biology: Exploring Life. Boston, MA: Pearson Prentice-Hall, 2006.

[29] https://commons.wikimedia.org/wiki/File:Popc_details.svg

[30] http://avantilipids.com/index.php?view=items \&cid=5\%3ALiposomes\&id=14\%3AWhat+Is+an+SUV+and+LUV+and+how+do+they+differ\%3F\&option=com_quickf aq\&Itemid $=385$

[31] https://en.wikipedia.org/wiki/Micelle

[32] http://medicine-opera.com/2009/07/trans-fats-big-brother-and-sloppy-science/

[33] https://dlc.dcccd.edu/biology1-3/lipids

[34] http://www.eufic.org/en/whats-in-food/article/facts-on-fats-the-basics

[35] Burr, G.O., Burr, M.M. and Miller, E. "On the nature and role of the fatty acids essential in nutrition". J. Biol. Chem. (1930). 86 (587).

[36] Linus, P., Corey, R. B., and Branson, H. R." The Structure of Proteins" Proc Natl Acad Sci U S A 37.4(1951): 205-11. 
[37] https://en.wikipedia.org/wiki/Lipid-anchored_protein

[38] Andrea, E.R. "Membrane proteins: always an insoluble problem?" Biochem Soc Trans 44.3 (2016): 790-95.

[39] Annela, M.S., Curnow, P., and Booth, P. J. "Membrane proteins, lipids and detergents: not just a soap opera" Biochimica et Biophysica Acta (BBA) Biomembranes 1666.1-2 (2004): 105-17.

[40] Elisabeth, P.C., Beis, K., Cameron, A.D., and Iwata, S. "Overcoming the challenges of membrane protein crystallography" Curr Opin Struct Biol 18.5 (2008): 581-86.

[41] Jonas, M.D., Scheidelaar, S., Koorengevel, M.C., Dominguez, J.J., Schäfer, M., van Walree, C.A., and Killian, J.A. " The styrene-maleic acid copolymer: a versatile tool in membrane research" Eur Biophys J 45(2016): 3-21.

[42] David, J.K.S., Scheidelaar, S., Foster, N., Grondelle, R., Killian, J.A., and Jones, M.R. "The effectiveness of styrene-maleic acid (SMA) copolymers for solubilisation of integral membrane proteins from SMA-accessible and SMAresistant membranes" Biochim Biophys Acta 1859.10 (2017): 2133-43.

[43] Xue, M., Cheng, L., Faustino, I., Guo, W., Marrink, S.J. "Molecular Mechanism of Lipid Nanodisk Formation by Styrene-Maleic Acid Copolymers" Biophys J. 115(2018): 494-502.

[44] Scheidelaar. S., Koorengevel. Martijn. C., Pardo,J., Meeldijk,J., Breukink, E., and Killian, J. "Molecular Model for the Solubilization of Membranes into Nanodisks by Styrene Malic Acid Copolymers" Biophysics Journal 108.2 (2015): 279-290.

[45] Pardo, Juan. J., Dörr, Jonas. M., Renne, M., Ould-Braham, T., Koorengevel, M., Steenbergen, M., and Killian, J." Thermotropic properties of phosphatidylcholine nanodiscs bounded by styrene-maleic acid copolymers" Membrane Biochemistry \& Biophysics, Bijvoet Center for Biomolecular Research 208 (2017): 58-64.

[46] Drummen, Gregor P. C. "Fluorescent Probes and Fluorescence (Microscopy) Techniques-Illuminating Biological and Biomedical Research" Molecules 17.12 (2012): 14067-14090.

[47] Veatch, S. L., O. Soubias, S. L. Keller, and K. Gawrisch. "Critical Fluctuations in Domain-forming Lipid Mixtures." Proceedings of the National Academy of Sciences 104.45 (2007): 17650-7655.

[48] Davis, James H., Jesse James Clair, and Janos Juhasz. "Phase Equilibria in DOPC/DPPC-d62/Cholesterol Mixtures." Biophysical Journal 96.2 (2009): 521-39.

[49] Shintzky, M., and Y. Barenholz. "Dynamics of the Hydrocarbon Layer in Liposomes of Lecithin and Sphingomyelin Containing Dicetylphosphate." The Journal of Biological Chemistry 249 (1974): 2652-657.

[50] http://www.interchim.fr/ft/1/12302A.pdf

[51] https://nptel.ac.in/courses/102103044/module2/lec6/1.html 
[52] Lakowicz, Joseph R. Principles of Fluorescence Spectroscopy. New York: Springer, 2006.

[53] Gratton, E., M. Limkeman, J.r. Lakowicz, B.p. Maliwal, H. Cherek, and G. Laczko. "Resolution of Mixtures of Fluorophores Using Variable-frequency Phase and Modulation Data." Biophysical Journal 46.4 (1984): 479-86.

[54] Lakowicz, Joseph R., Gabor Laczko, Ignacy Gryczynski, Henryk Szmacinski, and Wieslaw Wiczk. "New Trends in Photobiology." Journal of Photochemistry and Photobiology B: Biology 2.3 (1988): 295-311.

[55] www.jh-inst.cas.cz/ fluorescence/support/Lectures/UFCH_fluor03.pps

[56] https://link.springer.com/chapter/10.1007\%2F978-0-387-46312-4_10

[57] http://www.iss.com/resources/research/technical_notes/K2CH_AnisotropyDecay.html

[58] http://www.horiba.com/fileadmin/uploads/Scientific/Documents/Fluorescence/Tech_Note2_-_Anisotropy.pdf

[59] Blitterswijk, W. J. Van, R. P. Van Hoeven, and B.w W. Van Der Meer. "Lipid Structural Order Parameters (reciprocal of Fluidity) in Biomembranes Derived from Steady-state Fluorescence Polarization Measurements." Biochimica Et Biophysica Acta (BBA) - Biomembranes 644.2 (1981): 323-32.

[60] Mitchell, Drake C., and Burton J. Litman. "Molecular Order and Dynamics in Bilayers Consisting of Highly Polyunsaturated Phospholipids." Biophysical Journal 74.2 (1998): 879-91.

[61] Meer, W. Van Der, H. Pottel, W. Herreman, M. Ameloot, H. Hendrickx, and H. Schröder. "Effect of Orientational Order on the Decay of the Fluorescence Anisotropy in Membrane Suspensions. A New Approximate Solution of the Rotational Diffusion Equation." Biophysical Journal 46.4 (1984): 515-23.

[62] Szabo, Attila. "Theory of Fluorescence Depolarization in Macromolecules and Membranes." The Journal of Chemical Physics 81.1 (1984): 150.

[63] Meer, B.W. Van Der, R.p.h. Kooyman, and Y.k. Levine. "A Theory of Fluorescence Depolarization in Macroscopically Ordered Membrane Systems." Chemical Physics 66.1-2 (1982): 39-50.

[64] Pottel, H., W. Herreman, B.w. Van Der Meer, and M. Ameloot. "On the Significance of the Fourth-rank Orientational Order Parameter of Fluorophores in Membranes." Chemical Physics 102.1-2 (1986): 37-44.

[65] https://www.agilent.com/cs/library/usermanuals/Public/G111590042_OperatorManual.pdf

[66] https://www.chem.agilent.com/cag/prod/ca/lampimprove.pdf

[67] http://www.ecs.umass.edu/eve/facilities/equipment/Agilent8453resources.html

[68] https://www.wardsci.com/www.wardsci.com/images/BeerLamb_Colorimeter.pdf 
[69] http://www.iss.com/fluorescence/instruments/chronosFD.html

[70] Gratton, E, and Parasassi, T. "Fluorescence Lifetime Distributions in Membrane Systems" Journal of Fluorescence 5.1 (1995): 51-57.

[71] http://www.profoldin.com/sma21_for_nanodiscs.html

[72] Scheidelaar, S., Koorengevel, M., Walree, C., Dominguez, J., Dörr, J., and Killian, J. "Effect of Polymer Composition and $\mathrm{pH}$ on Membrane Solubilization by Styrene-Maleic Acid Copolymers" Biophysical Journal 111.9 (2016): 1974-1986. 